\title{
A Population of Faint Nontransient Low\#Mass Black Hole Binaries
}

\section{Citation}

Menou, Kristen, Ramesh Narayan, and Jean\#Pierre Lasota. 1999. "A Population of Faint Nontransient Low\#Mass Black Hole Binaries." The Astrophysical Journal 513 (2): 811-26. https://doi.org/10.1086/306878.

\section{Permanent link}

http://nrs.harvard.edu/urn-3:HUL.InstRepos:41384916

\section{Terms of Use}

This article was downloaded from Harvard University's DASH repository, and is made available under the terms and conditions applicable to Other Posted Material, as set forth at http:// nrs.harvard.edu/urn-3:HUL.InstRepos:dash.current.terms-of-use\#LAA

\section{Share Your Story}

The Harvard community has made this article openly available.

Please share how this access benefits you. Submit a story.

Accessibility 


\title{
A Population of Faint Non-Transient Low Mass Black Hole Binaries
}

\author{
Kristen Menou ${ }^{1}$ and Ramesh Narayan \\ Harvard-Smithsonian Center for Astrophysics, 60 Garden Street, \\ Cambridge, MA 02138, USA. \\ kmenou@cfa.harvard.edu,rnarayan@cfa.harvard.edu \\ and \\ Jean-Pierre Lasota \\ UPR 176 du CNRS, Département d'Astrophysique Relativiste et de Cosmologie, Observatoire de Paris, \\ Section de Meudon, 92195 Meudon Cédex, France. \\ lasota@obspm.fr
}

\begin{abstract}
We study the thermal and viscous stability of accretion flows in Low Mass Black Hole Binaries (LMBHBs). We consider a model in which an inner advection-dominated accretion flow (ADAF) is surrounded by a geometrically thin accretion disk, the transition between the two zones occurring at a radius $R_{\mathrm{tr}}$. In all the known LMBHBs, $R_{\mathrm{tr}}$ appears to be such that the outer disks could suffer from a global thermal-viscous instability. This instability is likely to cause the transient behavior of these systems. However, in most cases, if $R_{\mathrm{tr}}$ were slightly larger than the estimated values, the systems would be globally stable. This suggests that a population of faint persistent LMBHBs with globally stable outer disks could be present in the Galaxy. Such LMBHBs would be hard to detect because they would lack large amplitude outbursts, and because their ADAF zones would have very low radiative efficiencies, making the systems very dim. We present model spectra of such systems covering the optical and X-ray bands.
\end{abstract}

Subject headings: X-ray: stars - binaries: close - accretion, accretion disks - black hole physics - instabilities

\section{Introduction}

All known Low Mass Black Hole Binaries (LMBHBs) and several Low Mass Neutron Star Binaries (LMNSBs) are transient (see e.g. Tanaka \& Lewin 1995, van Paradijs \& McClintock 1995, White, Nagase \& Parmar 1995). These systems are also known as 'Soft X-ray Transients' or SXTs. In both LMBHBs and LMNSBs, a low mass secondary star (main sequence or sub-giant) transfers mass by Roche-lobe overflow to the compact primary. The transfered matter flows on to the primary via an accretion disk.

For effective temperatures between about $5000 \mathrm{~K}$ and $8000 \mathrm{~K}$, accretion disks described by the ' $\alpha$ viscosity' prescription are thermally and viscously unstable due to large changes in the opacity as a result of hydrogen recombination. This instability is believed to trigger outbursts of dwarf novae. A dwarf nova is a

\footnotetext{
1 also UPR 176 du CNRS, Département d'Astrophysique Relativiste et de Cosmologie, Observatoire de Paris, Section de Meudon, 92195 Meudon Cédex, France.
} 
cataclysmic binary (CB) system (Warner 1995) in which a white dwarf accretes from a low-mass companion at a mass accretion rate such that a steady disk would, at least over some range of radius, have an effective temperature in the thermally unstable range $5000-8000 \mathrm{~K}$. Since a steady disk is not allowed, the system undergoes a limit cycle where for some of the time (i.e during outburst) the mass accretion rate is high and the disk is entirely in a hot state with $T_{\text {eff }} \gtrsim 8000 \mathrm{~K}$, and for the rest of the time (during quiescence) the accretion rate is low and the entire disk is in a cold state with $T_{\text {eff }} \lesssim 5000 \mathrm{~K}$.

The disk instability model (DIM; see Cannizzo 1993 for a review and Hameury et al. 1998 for a recent version of the model) describes well the principal properties of dwarf novae. However, in its simplest version, the model cannot account for the variety of observed behaviors (e.g. Lasota \& Hameury 1998). In particular, the 'standard' version of the DIM cannot account for the properties of WZ Sge-type dwarf novae with large outburst amplitudes and long recurrence times. (Some of these systems are also called TOADs, but we will not use this name here.)

The WZ Sge-type dwarf novae resemble transient LMBHBs and LMNSBs (Lasota 1996a,b; Kuulkers, Howell \& van Paradijs 1996) and it was suggested quite early (van Paradijs \& Verbunt 1984; Cannizzo, Ghosh \& Wheeler 1995; Huang \& Wheeler 1989; Mineshige \& Wheeler 1989) that the same mechanism that triggers outbursts in dwarf novae also operates in transient LMBHBs and LMNSBs. However, detailed attempts to explain the properties of SXT outbursts (Mineshige \& Wheeler 1989) failed to reproduce the observed long recurrence times, which are of the order of 20 to 50 years in transient LMBHBs. Moreover, spectral observations of quiescent SXTs disagree seriously with the predictions of the DIM (Narayan, McClintock \& Yi 1996; Lasota 1996a). In particular, the observed X-ray emission in LMBHBs is more than four orders of magnitude larger than that predicted by the DIM. Not surprisingly similar problems are encountered in the dwarf nova case (Lasota 1996b).

Narayan et al. (1996) showed that the quiescent disk properties in SXTs could be explained with a model in which the accretion flow consists of two components: an outer CB-type thin accretion disk and an inner, advection-dominated accretion flow (ADAF) in which the radiative efficiency is very low. A similar model was proposed by Lasota, Hameury \& Huré (1995) for WZ Sge. There, however, the inner 'hole' in the CB accretion disk could be due to a weak magnetic field (Patterson et al. 1997). In the Narayan et al. (1996) model quiescent optical and UV radiation was emitted by the outer disk and the X-ray radiation came from the ADAF. The model, however, had a serious problem (Lasota, Narayan \& Yi 1996); some regions of the outer disk had effective temperatures within the unstable range, $5000 \mathrm{~K}<T_{\text {eff }}<8000 \mathrm{~K}$ (Wheeler 1996).

A modified version of the two-component model was subsequently proposed by Narayan, Barret \& McClintock (1997) which eliminates this problem. The transition radius is larger so that the outer disk is cool enough to be stable at all radii $\left(T_{\text {eff }}<5000 \mathrm{~K}\right)$. In this model, the optical and UV emission are from the ADAF and not the outer disk. The ADAF also produces the X-ray emission. The outer disk contributes only in the infrared, and even in this band its emission is overshadowed by the radiation from the secondary star.

It was noticed by Lasota et al. (1996) that the transition radii of SXTs, as determined from $\mathrm{H} \alpha$ line observations, are close to the values for which the outer thin disk would be globally stable. (By global stability, we mean that the disk can stably support a constant mass accretion rate; thus, the system will not undergo a limit cycle.) Based on this fact, Lasota et al. (1996) suggested that there may exist other closely related systems with a slightly larger transition radius in which the outer disk would, in fact, be globally stable. In the absence of significant variations of the mass-transfer rate such systems would not 
have transient outbursts, but would be steady and persistent. In addition, they would be extremely dim in $\mathrm{X}$-rays, and quite under-luminous even in the optical, so that they would be very difficult to detect. This 'hidden population' is the main subject of this paper.

For the remainder of the paper, we refer to optically thin two-temperature ADAFs simply as ADAFs (see Chen et al. 1995 for a distinction between various branches of accretion solutions). We refer to LMBHBs with a globally stable truncated thin disk (the topic of this paper) as faint persistent LMBHBs, or equivalently cold steady systems, and refer to the more standard transient LMBHBs with a globally unstable truncated thin disk as transient LMBHBs, or equivalently BH SXTs. The symbols $M, \dot{M}$ and $R$ refer to mass, mass accretion rate and radius in physical units, respectively, while the symbols $m, \dot{m}$ and $r$ refer to the same quantities in solar units $\left(m=M / M_{\odot}\right)$, in Eddindgton units $\left(\dot{m}=\dot{M} / \dot{M}_{\text {Edd }}\right.$, $\left.\dot{M}_{\text {Edd }}=1.39 \times 10^{18} \mathrm{~m} \mathrm{~g} \mathrm{~s}^{-1}\right)$ and in Schwarzschild units $\left(r=R / R_{S}, R_{S}=2.95 \times 10^{5} \mathrm{~m} \mathrm{~cm}\right)$.

\section{The Global Stability of LMBHB Accretion Flows}

We discuss the structure and stability of LMBHB accretion flows according to the model of quiescent SXTs developed by Narayan et al. (1997a). The accretion occurs primarily as a thin disk from the outer radius $r_{\text {out }}$ down to a transition radius $r_{\text {tr }}$, and then switches to a two-temperature ADAF for $r<r_{t r}$. The transition itself is not explained from first principles, but is introduced empirically.

This two-zone model successfully explains the observed properties of several SXTs in quiescence (Narayan et al. 1996, 1997a; Hameury et al. 1997b). Esin et al. (1997, 1998) show that such models also explain the various spectral states of black hole candidates, such as the high state, the intermediate state, and the low state, as sequences of varying accretion rate and location of the transition radius.

Lasota et al. (1996) discussed the mechanism for the origin of outbursts in BH SXTs and argued that the outbursts must be triggered by an instability in the outer thin disk component of the flow rather than in the inner ADAF zone. Two recent studies have strengthened the argument. First, Hameury et al. (1997b) have successfully reproduced all the properties of the rise to outburst of the BH SXT GRO J1655-40 with a model in which the outburst is triggered by the thermal-viscous instability of the outer disk. Second, detailed stability studies have confirmed that two-temperature ADAFs are essentially thermally and viscously stable (Kato et al. 1996, 1997; Wu, 1997\%). We therefore focus in this paper on the stability properties of the outer disk.

In the DIM, the stability criterion for a disk annulus can be expressed in terms of the local mass accretion rate $\dot{M}(R)$. If $\dot{M}(R)$ lies between two critical values, $\dot{M}_{\text {crit }}^{+}(R)$ and $\dot{M}_{\text {crit }}^{-}(R)$, then the effective temperature of the disk lies in the unstable range (5000-8000 K), and the local annulus is thermally and viscously unstable.

The disk is globally stable, and does not experience outbursts, whenever all its annuli are stable. This can be achieved in two ways. First, the disk can be in a hot, stable equilibrium if $\dot{M}(R)=$ constant $>\dot{M}_{\text {crit }}^{+}(R)$ for all radii $R$ in the disk. This is the standard scenario for persistent X-ray binaries and has been discussed recently by van Paradijs (1996), who included the effect of X-ray irradiation (but see also Dubus et al. 1998). Second, the disk can be in a cold, stable equilibrium if $\dot{M}(R)=$ constant $<\dot{M}_{\text {crit }}^{-}(R)$ at

\footnotetext{
${ }^{2}$ Note that $\mathrm{Wu}$ (1997) used vertically averaged equations to study the stability of the flow. Kato et al. (1997) have argued that this is incorrect.
} 
all $R$. It is the second option that we are interested in here.

Calculations of thin disk vertical equilibria (Hameury et al. 1998; see also Ludwig, Meyer-Hofmeister \& Ritter 1994) give

$$
\dot{M}_{\text {crit }}^{-}(R)=4.0 \times 10^{15} \alpha^{-0.04} m_{1}^{-0.89}\left(\frac{R}{10^{10} \mathrm{~cm}}\right)^{2.67} \mathrm{~g} \mathrm{~s}^{-1}
$$

where $\alpha \leq 1$ is the turbulent viscosity parameter (Shakura \& Sunyaev 1973), $m_{1}$ is the mass of the accreting primary in solar mass units, and $R$ is the radius of the annulus under consideration. Since $\dot{M}_{\text {crit }}^{-}(R)$ is a steep function of $R$, we are unlikely to find a system with a cold, stable equilibrium if the thin accretion disk extends all the way down to the last stable orbit around a black hole. The limit on $\dot{M}$ in this case is so low (of order $10^{6} \mathrm{~g} \mathrm{~s}^{-1}$ typically) that any system that satisfies the constraint $\dot{M}<\dot{M}_{\text {crit }}^{-}\left(R_{\text {in }}\right)$ would be observationally very uninteresting (this statement is true even if we use very low values of $\alpha$, e.g. $10^{-4}$ ). However, if the disk is truncated at a radius that is significantly outside the marginally stable orbit, then it is possible to have a globally stable cold flow with reasonable values of $\dot{M}$.

Eq. (1) shows that for a given, constant, accretion rate $\dot{M}$ there exists a critical radius $R_{\text {crit }}$ given by

$$
R_{\text {crit }}=6 \times 10^{9}\left(\frac{\dot{M}}{10^{15} \mathrm{~g} \mathrm{~s}^{-1}}\right)^{0.375} \alpha^{0.015} m_{1}^{0.33} \mathrm{~cm} .
$$

If the transition from the outer disk to the ADAF occurs at a radius $R_{\text {tr }}$ greater than this $R_{\text {crit }}$, the flow will be cold and globally stable.

In two-component accretion flows, the transition between the thin disk and the ADAF most likely occurs over a range of radius by the gradual evaporation of the thin disk material (Meyer \& Meyer-Hofmeister 1994; Narayan \& Yi 1995). The transition is thus not abrupt, although the width of the transition region is likely to be narrow (Esin et al. 1997; Abramowicz et al. 1998). We assume for the remainder of this work that the evaporation process does not affect the disk stability. This is a reasonable approximation if the evaporation law is steep (for example, the $1 / r$ law for the coronal accretion rate used by Esin et al. 1997) since the accretion rate in the thin disk is then almost unchanged except very close to the transition radius. Note that evaporation reduces the accretion rate in the disk. According to Eq. (1), it should therefore increase the stability of the disk.

\subsection{Prescription for the Transition Radius $\mathbf{R}_{\mathrm{tr}}$}

The location of $R_{\mathrm{tr}}$ is ultimately determined by the physics of the evaporation of gas from the thin accretion disk to the ADAF. This physics is poorly understood. Several mechanisms have been proposed in the literature (Meyer \& Meyer-Hofmeister 1994, Narayan \& Yi 1995, Honma 1996) to explain the transition, but none of these models has reached a sufficient level of detail to allow quantitative predictions (see e.g. Narayan, Mahadevan \& Quataert 1998 for a discussion).

Quite independent of the actual process of evaporation, various limits can be derived on the value of $R_{\mathrm{tr}}$. First, the dynamics of the incoming stream from the secondary leads to a maximum value of $R_{\mathrm{tr}}$. At the same time, observations of the $H_{\alpha}$ line from the disk provide an independent limit on $R_{\mathrm{tr}}$. These two constraints are consistent with each other, as we show in $\S 2.1 .1$ and $\S 2.1 .2$. Another limit arises from the 
fact that for a given mass accretion rate, an ADAF is not allowed beyond a certain maximum radius $R_{\max }$. We estimate this limit in $§ 2.1 .3$.

These arguments give only upper limits on $R_{\mathrm{tr}}$. In this paper we assume that the ADAF extends out to the largest radius it is allowed (Narayan \& Yi 1995), that is to the smallest of the various upper limits. Observations of quiescent SXTs are consistent with this assumption, as we discuss in $§ 2.1 .4$, and we assume that the systems we discuss in this paper also satisfy this rule. However, we note that the assumption is apparently not satisfied in the nucleus of NGC 4258 (Gammie, Blandford \& Narayan 1998).

\subsubsection{Constraint from Stream Dynamics}

According to the calculations of Lubow \& Shu (1976) and Lubow (1989), a substantial fraction of the stream of matter from the secondary overflows the rim of the accretion disk and impacts at a radius $R_{\text {impact }}$ that is significantly smaller than the outer radius of the disk, $R_{\text {out }}$. Such overflows are seen in 'dipping' low mass X-ray binaries (see e.g. Uno et al. 1997; Frank, King \& Lasota 1987) and in cataclysmic variables (see Warner 1995).

We do not expect the ADAF zone to extend to radii larger than the impact radius of the stream. This is because the cold stream moves very rapidly (essentially at free-fall speed) from the Roche lobe of the secondary to the impact point; it is unlikely that the material in the stream could evaporate into an ADAF in this short time. At the very least, we expect the cold incoming stream to form an annulus at the impact radius $R_{\text {impact }}$. More likely, the material will spread a little under the influence of viscous evolution before it evaporates fully. Thus we expect $R_{\mathrm{tr}} \lesssim R_{\text {impact }}$.

Lubow's calculations show that, in the free-stream approximation, all overflowing streams converge to a single impact radius which scales to a good approximation as $R_{\text {impact }} \simeq 0.48 R_{\text {circ }}$ (the coefficient corresponds to a low mass ratio $q=m_{2} / m_{1}<0.5$, which is valid for all the systems we consider). Here, $R_{\text {circ }}$ is the circularization radius of the binary (Frank et al. 1992),

$$
\begin{aligned}
\frac{R_{\text {circ }}}{a} & =(1+q)[0.5-0.227 \times \log q]^{4} \\
a & =3.5 \times 10^{10} m_{1}^{1 / 3}(1+q)^{1 / 3} P_{\mathrm{hr}}^{2 / 3} \mathrm{~cm}
\end{aligned}
$$

where $a$ is the orbital separation of the binary, and $P_{\mathrm{hr}}$ is the orbital period in hours. Since we have argued that $R_{\text {impact }}$ is an upper limit on the transition radius, we write

$$
R_{\mathrm{tr}}=f_{t} R_{\mathrm{circ}}, \quad f_{t}<0.48
$$

The systems that we consider span almost two orders of magnitude in orbital period. Since the circularization radius varies as $P_{\text {orb }}^{2 / 3}$, the impact radius also spans a wide range. In principle, the parameter $f_{t}$ may vary in some complicated fashion as a function of $R_{\text {impact }}$. For simplicity, we treat $f_{t}$ as a free parameter, which absorbs all the complicated physics associated with the stream dynamics, and consider values between 0.1 and 0.48 . 


\subsubsection{Constraint from $H_{\alpha}$ Emission Line Width}

Observations of $H_{\alpha}$ emission lines from the disk constrain the value of $R_{\mathrm{tr}}$ in quiescent BH SXTs. As emphasized by Narayan et al. (1996), half the full-width at zero-intensity of the $H_{\alpha}$ emission line provides a good lower limit to the speed of matter, $v_{\text {tr }}$, at the transition radius. This can be translated into an upper limit on the transition radius through the relation (assuming a Keplerian disk)

$$
r_{\mathrm{tr}}<\frac{1}{2}\left(\frac{c \sin i}{v_{\mathrm{tr}}}\right)^{2}
$$

where $c$ is the speed of light and $i$ is the inclination of the binary system to the line of sight. Narayan et al. (1996) used this relation to derive upper limits on $r_{\text {tr }}$ in the SXTs A0620-00, V404 Cyg and Nova Mus 91, and Hameury et al. (1997b) did the same for GRO J1655-40. We derive upper limits for three other quiescent systems, GRO J0422+32, GS2000+25 and Nova Oph 77, using the inclinations summarized in Table 1 and the following lower limits on $v_{\mathrm{tr}}$, collected from the literature: $v_{\mathrm{tr}}>1000 \mathrm{~km} \mathrm{~s}^{-1}$ in GRO J0422+32 (Garcia et al. 1996, Orosz \& Bailyn 1995), $v_{\mathrm{tr}}>1400 \mathrm{~km} \mathrm{~s}^{-1}$ in GS2000+25 (Harlaftis et al. 1996) and $v_{\mathrm{tr}}>1000 \mathrm{~km} \mathrm{~s}^{-1}$ in Nova Oph. 77 (Harlaftis et al. 1997). The upper limits on $r_{\mathrm{tr}}$ for all seven systems are indicated by downward arrows in Fig. 1.

Figure 11 also shows the predictions for the transition radius according to Eq. (5). Four values of the parameter $f_{t}$ are considered: $f_{t}=0.1,0.2,0.3,0.48$. Note that the circularization radius and hence the impact radius in a binary system depends on the masses of the stars. Therefore, a knowledge of the masses as functions of the orbital period of the system is required in order to use Eq. (5). The mass of the secondary at a given period is theoretically constrained by binary evolution theory. Semi-detached binaries in which main-sequence donors fill their Roche-lobes satisfy the relation $m_{2} \approx 0.11 P_{\mathrm{hr}}$, where $P_{\mathrm{hr}}$ is the orbital period of the system in hours (e.g. Frank et al. 1992). When the secondary is a subgiant or a giant, the period mass relation is less simple because it depends also on the core mass of the star (e.g. King 1988). For simplicity, in Fig. 11 we have assumed that we have 'typical' systems with $m_{1}=7$ and $m_{2}=\min \left[0.11 P_{\mathrm{hr}}, 1\right]$. This assumption is valid for four of the SXTs listed in Table 1. The systems J0422+32, J1655-40 and V404 Cyg deviate somewhat from this assumption. We have derived values of $R_{\mathrm{tr}}$ for these systems, taking into account their actual masses; the results are not very different.

Technically, the $H_{\alpha}$ line widths only give upper limits on the transition radius; the fact that $H_{\alpha}$ emission is not observed from gas orbiting at higher speeds may merely mean that such high velocity material does not emit enough radiation to be detectable (small emitting area). We make the stronger assumption that the high velocity material is absent altogether in the cold disk, so that the $H_{\alpha}$ line widths directly give an estimate of $R_{\mathrm{tr}}$ to within a factor of a few. In that case, we see that most of the systems shown in Fig. 1 are consistent with $f_{t} \sim 0.25$. More precise calculations of the circularization radii of J0422+32, J1655-40 and V404 Cyg, taking into account $m_{1}$ and $m_{2}$ in Table 1 , show that the first two of these systems are also in general agreement with the condition $f_{t} \sim 0.25$. The only peculiar object is V404 Cyg, which appears to require $f_{t}<0.1$. This system, however, has the longest orbital period and may

behave differently because of this (see §2.1.3). Życki, Done \& Smith (1998) have also argued for a low value of the transition radius in V404 Cyg. 


\subsubsection{Maximum Radius for the ADAF}

At each radius $r$ in the accretion flow, there is a critical accretion rate $\dot{m}_{\text {crit }}$ above which it is not possible to have an ADAF solution (see Lasota 1998 for a recent discussion). This critical $\dot{m}_{\text {crit }}$ was estimated by Abramowicz et al. (1995) using only bremsstrahlung cooling, by Narayan \& Yi (1995) using a more detailed model of cooling, and more recently by Esin (1997) and Esin et al. (1997). We present an improved calculation here.

We are primarily interested in large radii, $r \gtrsim 10^{3.5}$, where the plasma in the ADAF is essentially one-temperature (Narayan \& Yi 1995). We therefore set the ion and electron temperatures equal. We also assume that the flow is described well by the self-similar solution of Narayan \& Yi (1994), which is

well-supported by the detailed global studies of Narayan, Kato \& Honma (1997) and Chen, Abramowicz \& Lasota (1997). The electron density and temperature of the gas in the ADAF are then given by (Narayan \& Yi 1995)

$$
\begin{aligned}
n_{e} & =2.00 \times 10^{19} \alpha_{\mathrm{ADAF}}^{-1} c_{1}^{-1} c_{3}^{-1 / 2} m^{-1} \dot{m} r^{-3 / 2} \mathrm{~cm}^{-3}, \\
T & =3.20 \times 10^{12} \beta c_{3} r^{-1} \mathrm{~K} .
\end{aligned}
$$

Here $\alpha_{\mathrm{ADAF}}$ is the usual $\alpha$ viscosity parameter, as defined for the ADAF, and $\beta$ is the ratio of gas to total pressure, which we set to 0.5 (perfect equipartition). The parameters $c_{1}$ and $c_{3}$ are defined to be

$$
\begin{aligned}
& c_{1}=\frac{5+2 \epsilon^{\prime}}{3 \alpha_{\mathrm{ADAF}}^{2}} g\left(\alpha_{\mathrm{ADAF}}, \epsilon^{\prime}\right), \quad c_{3}=\frac{2\left(5+2 \epsilon^{\prime}\right)}{9 \alpha_{\mathrm{ADAF}}^{2}} g\left(\alpha_{\mathrm{ADAF}}, \epsilon^{\prime}\right), \\
& g\left(\alpha_{\mathrm{ADAF}}, \epsilon^{\prime}\right)=\left[1+\frac{18 \alpha_{\mathrm{ADAF}}^{2}}{\left(5+2 \epsilon^{\prime}\right)^{2}}\right]^{1 / 2}-1, \\
& \epsilon^{\prime}=\frac{1}{f_{\mathrm{adv}}}\left(\frac{5 / 3-\gamma}{\gamma-1}\right) .
\end{aligned}
$$

Here $\gamma$ is the adiabatic index of the gas which, following Esin (1997), we set to

$$
\gamma=\frac{8-3 \beta}{6-3 \beta}=1.4444 \quad(\text { for } \beta=0.5)
$$

Finally, $f_{\text {adv }}$ is the advection parameter of the accretion flow, defined as the fraction of the viscous heat energy that is advected with the gas.

Given the values of $m, \dot{m}, r, \alpha_{\mathrm{ADAF}}$ and $\beta(=0.5)$, the values of $n_{e}$ and $T$ depend only on the advection parameter $f_{\text {adv }}$. For the moment, let us treat $f_{\text {adv }}$ as a free parameter. The self-similar solution then gives the volume heating rate due to viscous dissipation to be

$$
q^{+}=1.84 \times 10^{21} \epsilon^{\prime} c_{3}^{1 / 2} m^{-2} \dot{m} r^{-4} \mathrm{erg} \mathrm{cm}^{-3} \mathrm{~s}^{-1},
$$

and the cooling rate to be

$$
q^{-}=n_{e}^{2} \Lambda(T)=4.00 \times 10^{38} \epsilon^{\prime} \alpha_{\mathrm{ADAF}}^{-2} c_{1}^{-2} c_{3}^{-1} m^{-2} \dot{m}^{2} r^{-3} \Lambda(T) \operatorname{erg} \mathrm{cm}^{-3} \mathrm{~s}^{-1},
$$

where $n_{e}^{2} \Lambda(T)$ is the cooling rate per unit volume of the hot optically thin gas. For our calculations, we use a standard $\Lambda(T)$, shown in Fig. 2a, which is similar to the curve shown in Dalgarno \& McCray (1972). 
The free parameter in the above expressions is $f_{\text {adv }}$. We now determine this parameter self-consistently. Since $f_{\text {adv }}$ is the fraction of the viscous energy that is advected, we can equally well say that $\left(1-f_{\text {adv }}\right)$ is the fraction of energy that is radiated. (We are ignoring compressive heating, which is a reasonable approximation at the mass accretion rates that we are considering; see Nakamura et al. 1997, Mahadevan \& Quataert 1997.) Thus, we determine $f_{\text {adv }}$ by solving

$$
q^{-}=\left(1-f_{\text {adv }}\right) q^{+} .
$$

Equivalently, we can solve for $\dot{m}$ as a function of $f_{\text {adv }}$ :

$$
\dot{m}=\frac{4.6 \times 10^{-18}}{\Lambda(T)} \alpha_{\mathrm{ADAF}}^{2} \epsilon^{\prime} c_{1}^{2} c_{3}^{3 / 2} r^{-1}\left(1-f_{\mathrm{adv}}\right) .
$$

This relation gives a mapping between $\dot{m}$ and $f_{\text {adv }}$. Note that $\dot{m}$ is independent of $m$. Note also that if the cooling is simply by bremsstrahlung, $\Lambda(T) \propto T^{1 / 2} \propto r^{-1 / 2}$. We then expect $\dot{m}_{\text {crit }} \propto r^{-1 / 2}$. This dependence was derived by Abramowicz et al. (1995) and Narayan \& Yi (1995). We use a more realistic $\Lambda(T)$ and obtain a different $r$ dependence of $\dot{m}_{\text {crit }}$.

Figure 2 $2 \mathrm{~b}$ shows curves of $\dot{m}$ vs $f_{\mathrm{adv}}$ for $\alpha_{\mathrm{ADAF}}=0.3, \beta=0.5$, and various radii $r$. At each $r$, we see that there is a maximum $\dot{m}$ up to which solutions for $f_{\text {adv }}$ exist, but above which there is no solution at all. (Compare with a similar curve shown in Fig. 6a of Esin et al. 1997). The maximum $\dot{m}$ is what we call $\dot{m}_{\text {crit }}$. For $\dot{m}<\dot{m}_{\text {crit }}$, there are two solutions for $f_{\text {adv }}$. The solution with the larger $f_{\text {adv }}$ (i.e. advection-dominated) is the stable ADAF branch (Narayan \& Yi 1995). The solution with the smaller $f_{\text {adv }}$ (i.e. cooling-dominated) is the SLE branch (Shapiro, Lightman \& Eardley 1976) which is unstable (Piran 1978; Narayan \& Yi 1995).

Figure 3 shows $\dot{m}_{\text {crit }}$ vs $r$ for two different values of $\alpha_{\text {ADAF }}$. The wiggles in the curves are the result of the various features in the cooling function $\Lambda(T)$. Leaving the wiggles aside, we see that $\dot{m}_{\text {crit }}$ decreases monotically with increasing $r$. Now, Fig. 3 could equally well be described as a plot of the maximum radius $r_{\max }$ out to which an ADAF is allowed for a given $\dot{m}$. In other words, the horizontal axis could be interpreted as showing $r_{\max }$ as a function of $\dot{m}$ along the vertical axis. We see that $r_{\max }(\dot{m})$ is a monotically increasing function of decreasing $\dot{m}$. For a given $\dot{m}$, a pure ADAF flow is allowed only for $r<r_{\max }(\dot{m})$; beyond $r_{\max }(\dot{m})$ a thin disk plus an ADAF-like corona is likely to be present (cf Esin et al. 1997).

\subsubsection{Complete Prescription for $r_{\mathrm{tr}}$}

We now combine the ideas described in $\S 2.1 .1$ and 2.1.3. Figure 4 shows the results for a LMBHB with $\alpha_{\mathrm{ADAF}}=0.3, m_{1}=7, m_{2}=\min \left[0.11 P_{\mathrm{hr}}, 1\right]$ and the choice $f_{t}=0.2$. The abscissa shows the transition radius $r_{\text {tr }}$ and the ordinate shows the mass accretion rate $\dot{M}$ in the accretion flow. When $\dot{M}$ is low, the transition radius is determined by stream dynamics (\$2.1.1) and is a function only of the orbital period of the binary. This is shown by the three vertical dotted lines which correspond to $P_{\text {orb }}=5 \mathrm{hr}, 20 \mathrm{hr}$ and 100 hr, respectively.

However, at each value of $P_{\text {orb }}$, there is a critical $\dot{M}$ above which an ADAF is not allowed at the value of $r_{\mathrm{tr}}$ determined by stream dynamics. At such high values of $\dot{M}$, the thin disk has to extend down to a smaller radius, namely to the radius $r_{\max }$ calculated in $\S 2.1 .3$ and shown by the solid line in Fig. 1 . The critical $\dot{M}$ at which $r_{\text {tr }}$ switches from the value given by stream dynamics to $r_{\max }$ differs for different orbital periods, as seen from Fig. 4 . 


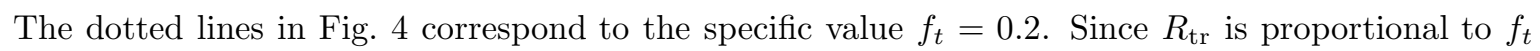
(Eq. (5)), increasing or decreasing $f_{t}$ would shift the dotted lines to the right or left, respectively.

\subsection{A New Criterion for the Global Stability of LMBHBs}

According to the DIM, the accretion disk of a LMBHB is stable if the critical radius $r_{\text {crit }}$ of the system (Eq. (2)) is smaller than the transition radius $r_{\text {tr }}$. Figure 5 is similar to Fig. 目 but with an additional dashed line which shows the location of $r_{\text {crit }}$ as a function of $\dot{M}$. Any system that lies to the right of the dashed line is stable, while any system to the left of the dashed line is unstable.

The open circles in Fig. 5 show cases of marginal stability for three orbital periods. The lowermost circle shows the maximum $\dot{M}$ up to which a system with an orbital period of $5 \mathrm{hr}$ would be globally stable. The middle circle is the corresponding maximum $\dot{M}$ for a $20 \mathrm{hr}$ binary. The upper circle, which corresponds to $P_{\text {orb }} \sim 70 \mathrm{hr}, \dot{M}=10^{17} \mathrm{~g} \mathrm{~s}^{-1}$, is a critical case. This is the limiting $\dot{M}$ for all systems with $P_{\text {orb }} \geq 70 \mathrm{hr}$. In all such systems, the transition radius $r_{\mathrm{tr}}$ is determined, not by stream dynamics, but by the limiting value $r_{\max }$. At accretion rates $\dot{M}>10^{17} \mathrm{~g} \mathrm{~s}^{-1}$ (for the set of parameters chosen in Fig. D), a LMBHB, whatever its period, is unstable because its transition radius is necessarily to the left of the dashed line.

The above considerations can be summarized by the simple criterion shown in Fig. 6, which corresponds to the set of parameters used in Figs. 1 and 5 . A LMBHB with a given orbital period is unstable if its mass transfer rate $\dot{M}_{T}$ is above the solid line and stable if $\dot{M}_{T}$ is below this line (for $\alpha_{\mathrm{ADAF}}=0.3$ ). If the system is stable, it will be a faint persistent LMBHB and very difficult to detect. The break in the solid line at $P_{\text {orb }} \sim 70 \mathrm{hr}$ occurs because for $P_{\text {orb }}>70 \mathrm{hr}$ the transition radius $r_{\mathrm{tr}}$ is determined by $r_{\text {max }}$ rather than by stream dynamics. The second break at $\sim 10 \mathrm{hr}$ corresponds to the transition from $m_{2}=0.11 P_{\mathrm{hr}}$ to $m_{2}=1$. Note that faint persistent systems are more likely to exist at large orbital periods because the stability criterion is less stringent there.

\section{Comparison with Observed Transient Systems}

The purpose of this section is to establish that transient systems could be limited to a fairly narrow instability band in the $P_{\text {orb }}-\dot{M}$ plane. Outside this band, the systems would be either hot and steady (at high accretion rates) or cold and steady (at low accretion rates).

We also establish that known transient systems are all found within the instability band. This test requires a knowledge of the orbital periods and mass accretion rates of the various BH SXTs. The orbital periods of the seven BH SXTs of interest $[$ are well known and are listed in Table 1. The accretion rates in the systems are, however, more uncertain and are necessarily model-dependent. We describe below the method we use to estimate $\dot{M}$.

\footnotetext{
${ }^{3}$ We do not consider $4 \mathrm{U}$ 1543-47 because there is insufficient spectral data on this newly established BH SXT (Orosz et al. 1998).
} 


\subsection{Accretion Rates in BH SXTs}

The standard procedure to estimate the average mass accretion rate in a SXT is based on the idea that SXTs mainly store mass during quiescence and accrete it suddenly during an outburst (e.g. McClintock et al. 1983, van Paradijs 1996). The rate at which mass accumulates in the disk, $\dot{M}_{\text {accum }}$, can be estimated by measuring the integrated flux from the system during an outburst (§3.1.1). This is a reliable method since there is good evidence that SXTs accrete via a thin disk in outburst (e.g. Tanaka \& Shibazaki 1996), so that the radiative efficiency during outburst is well understood. However, the successful application of ADAF models to BH SXTs in quiescence (Narayan, Barret \& McClintock, 1997) shows that these systems accrete substantial amounts of mass even during quiescence via an inefficiently radiating ADAF (§3.1.2). Therefore, the total accretion rate in a system is the sum

$$
\dot{M}_{\text {tot }}=\dot{M}_{\text {accum }}+\dot{M}_{\mathrm{ADAF}},
$$

where $\dot{M}_{\mathrm{ADAF}}$ is the accretion rate during quiescence (assumed to be constant over the inter-outburst time). The total mass accretion rate, $\dot{M}_{\text {tot }}$, must be equal to the mass transfer rate from the secondary, $\dot{M}_{T}$, provided all the transferred mass is accreted by the primary. In what follows, we derive $\dot{M}_{\text {accum }}$ and $\dot{M}_{\mathrm{ADAF}}$ for the seven BH SXTs listed in Table 1 .

\subsubsection{Mass Accumulation Rates}

The accumulation rate $\dot{M}_{\text {accum }}$ can be deduced from the total fluence, $\Delta F$, of one of its outbursts and the inter-outburst time, $\Delta t$ :

$$
\dot{M}_{\text {accum }}=\frac{\Delta F}{\Delta t \eta c^{2}},
$$

where $c$ is the speed of light and $\eta$ is the radiative efficiency of accretion during outburst. We assume $\eta=0.1$, which is appropriate for a normal thin accretion disk around a Schwarzschild or slowly spinning black hole.

The observational data and the system parameters that we use to estimate the accumulation mass accretion rates are listed in Table 15. Our data are a little different (mainly distances and outburst fluences) from those used by van Paradijs (1996). For A0620-00 and V404 Cyg, more than one outburst has been observed, giving $\Delta t$, so that firm estimates for $\dot{M}_{\text {accum }}$ may be derived. For the other systems, only upper

limits to $\dot{M}_{\text {accum }}$ are deduced because we have only lower limits on $\Delta t$. The full set of estimates for $\dot{M}_{\text {accum }}$ are listed in Table 3. Our estimate for A0620-00 is in good agreement with the estimate of McClintock et al. (1983).

\subsubsection{Accretion Rates in ADAFs}

We estimate the ADAF accretion rates in quiescence, $\dot{M}_{\mathrm{ADAF}}$, by fitting ADAF spectral models to the observational data for each system. Note that the outer thin disks do not contribute significantly to the quiescent spectra of the systems we consider so that the results are insensitive to the precise choice of the transition radius.

Spectral models of A0620-00, V404 Cyg and Nova Mus 91 were constructed by Narayan et al. (1996, 1997a), and J1655-40 was similarly modeled by Hameury et al. (1997b). We extend the previous work on 
these systems using an improved version of the ADAF model. We also construct new ADAF models of GRO J0422+32, GS2000+25 and Nova Oph 77 in quiescence.

The new version of the ADAF model is described in Narayan et al. (1998). One of the main improvements relative to older versions is that the model now consistently includes adiabatic compressive heating of electrons in the energy equation (Nakamura et al. 1997). It also uses for the flow dynamics the general relativistic global solutions calculated by Popham \& Gammie (1998).

The modeling technique used in previous studies was to fit the quiescent X-ray flux by adjusting $\dot{M}_{\mathrm{ADAF}}$, keeping the other model parameters fixed at the following canonical values (see Narayan, Mahadevan \& Quataert 1998 for a discussion): $\alpha_{\mathrm{ADAF}}=0.3, \beta=0.5, \gamma=1.4444$ (see Eq. (12)). We use the same modeling technique here to estimate $\dot{M}_{\mathrm{ADAF}}$ in those systems for which there is an X-ray flux in quiescence. In the remaining systems, we fit the models to optical data. The estimates of $\dot{M}_{\mathrm{ADAF}}$ are given in Table 3 and the spectral fits are shown in Figs. 7 and 8. Note that the estimates of $\dot{M}_{\mathrm{ADAF}}$ would be reduced if $\alpha_{\mathrm{ADAF}}$ was reduced, for instance, from 0.3 to 0.1 .

For A0620-00, V404 Cyg, and GRO J1655-40 we used the observational data summarized by Narayan et al. (1996, 1997a) and Hameury et al. (1997b) and fitted the X-ray fluxes (solid lines, Fig. (7). We also constructed models which fit the optical data points (dashed lines, Fig 7 ) to get some idea of the error in the estimate of $\dot{M}_{\mathrm{ADAF}}$. The resulting estimates are listed in Table 3 . Note that in GRO J1655-40, the "disk" contributes only $\sim 5 \%$ of the total optical flux (dominated by the $F$-type secondary), so that the constraint from the optical data is rather weak (Hameury et al. 1997b).

The remaining systems have only X-ray upper limits in quiescence and we are limited to fitting the optical data. For Nova Mus 91 we used the optical data summarized in Narayan et al. (1996). For the other three systems, namely GRO J0422+32, GS2000+25 and Nova Oph 77, we collected optical data from the literature. Due to the faintness of the sources, the emission in the optical is somewhat poorly constrained. We derived the net optical fluxes due to accretion, by applying a correction for interstellar extinction at the wavelength of observation (with a standard proportionality constant between the extinction and the color excess $R_{V}=3.1$; Cardelli, Clayton \& Mathis 1989) and subtracting the estimated flux of the secondary. The net optical fluxes that we deduce in these systems are listed in Table 2. For GRO J0422+32 and GS2000+25, the uncertainty comes mainly from the contamination of the secondary, while for Nova Oph 77 the uncertainty is primarily from the intrinsic variability of the source.

The ADAF spectral models for these four sources are shown in Fig. 8, for a range of accretion rates which cover the uncertainties in the net optical fluxes. Note that all the models satisfy the X-ray upper limits quite comfortably. We summarize in Table 3 the estimates for $\dot{M}_{\mathrm{ADAF}}$ inferred from the spectral models.

\subsubsection{Total Mass Accretion Rates}

The last column in Table 3 gives our estimates of $\dot{M}_{\text {tot }}$, namely the sum of $\dot{M}_{\text {accum }}$ and $\dot{M}_{\mathrm{ADAF}}$. An arbitrary factor of 1.5 has been applied to the lower and upper bounds of the estimates to reflect the fact that we do not trust our estimates to an accuracy better than a factor 2 typically. A look at Table 3 shows that in most systems, $\dot{M}_{\mathrm{ADAF}}$ is comparable to or greater than $\dot{M}_{\text {accum }}$ (for which we have mainly upper limits), so that $\dot{M}_{\text {tot }}$ is effectively a firm estimate. For Nova Mus 91 and GS2000+25, in which $\dot{M}_{\text {accum }}>\dot{M}_{\mathrm{ADAF}}$, only upper limits to $\dot{M}_{\text {tot }}$ are deduced. Note, however, that $\dot{M}_{\text {tot }}$ cannot go below 
$\dot{M}_{\mathrm{ADAF}}$, which is $\sim 2 \times 10^{15} \mathrm{~g} \mathrm{~s}^{-1}$ in these two systems.

Our estimates of $\dot{M}_{\text {tot }}$ are in good agreement with the evolutionary calculations of Pylyser \& Savonije (1988) for A0620-00 and King (1993) for V404 Cyg (closer to his maximum mass solution). Several complications may affect our result for J1655-40. The source remained a persistent X-ray source for several months after its April 1996 outburst (e.g. Hynes et al. 1998) and this has not been taken into account in the fluences listed in Table 1 and used to estimate $\dot{M}_{\text {accum }}$ (Chen et al. 1997). This could explain, at least in part, the relatively low estimate obtained for $\dot{M}_{\text {accum }}$ (see Table 31). The evolutionary status of J1655-40 is also very peculiar (see e.g. Kolb 1998). In addition, Zhang, Cui \& Chen (1997) have argued that the central black hole in J1655-40 is a Kerr black hole in fast prograde rotation, so that the efficiency of accretion in the system is more likely to be $\eta \sim 0.4$ rather than 0.1 . Taking this into account would

reduce $\dot{M}_{\text {accum }}$ even more in this system. Finally, the mass loss from the secondary could be related to the jet activity in this source (Hjellming \& Rupen 1995, King - private communication) which would make it difficult to get an independent estimate of $\dot{M}_{\text {tot }}$.

\subsection{On the Stability of Known BH SXTs}

Four of the seven BH SXTs listed in Table 1, namely A0620-00, GS2000+25, Nova Mus 91 and Nova Oph 77, have mass characteristics relatively close to those of a 'typical' system with $m_{1}=7$ and $m_{2}=\min \left[0.11 P_{\mathrm{hr}}, 1\right]$. The estimated ranges of $\dot{M}_{\text {tot }}$ in these four systems are shown in Fig. 9 as errorbars in the $P_{\text {orb }}-\dot{M}_{T}$ plane. The three solid lines are stability criteria as in Fig. 6 but for three choices of the stream dynamics parameter, $f_{t}=0.3,0.2,0.1(\S 2.1 .4)$.

Observations of $H_{\alpha}$ emission lines imply that transition radii have to be relatively small $\left(f_{t} \lesssim 0.2-0.25\right)$ in quiescent BH SXTs (§2.1.2). Figure 9 shows that for such values of $f_{t}$, the four systems are indeed unstable according to the stability criterion. The estimates of $\dot{M}_{\text {tot }}$ in GS2000+25 and Nova Mus 91 are subject to uncertainty and may well go down. However, they will not do so by more than an order of magnitude (cf §3.1.3). Therefore, these systems are likely to remain in the unstable zone. For the three other BH SXTs, namely J0422+32, J1655-40 and V404 Cyg, the stability criteria have to be computed for their specific mass characteristics. We find that J0422+32 and J1655-40 are unstable, so long as $f_{t} \lesssim 0.25$, although the evidence is less strong than in the previous four cases (the estimates of $\dot{M}_{\text {tot }}$ are not firmly above the stability line corresponding to $f_{t}=0.2$ ). This could be explained in J0422+32 by the fact that the mass of the central black hole, which has a substantial effect on the stability criterion, is not well known (Beekman et al. 1997; we take $m_{1}=12$ ). In the case of J1655-40, we expect $\dot{M}_{\text {tot }}$ to be larger than our estimate (because some activity was not taken into account, cf $\S 3.1 .3$ ), which could easily drive the system firmly into the region of instability. Our analysis shows that V404 Cyg would be stable if $f_{t}=0.2$ and unstable if $f_{t} \lesssim 0.1$. This is consistent with the maximum value for $f_{t}$ deduced from observations of this system $(\S 2.1 .2)$.

These results strongly suggest that the truncated disks found in BH SXTs are unstable and that the systems will undergo the thermal-viscous instability. All of these systems are indeed transients and do show large variability. Thus the same mechanism could drive outbursts of both DNe and SXTs (van Paradijs \& Verbunt 1984). It is not possible, however, to firmly rule out the possibility that at least some of the disks are stable since the systems generally lie close to the stability region. In this case SXT outbursts could be triggered by variations in the mass-transfer rate (but outbursts would still be due to a thermal-viscous instability) as proposed by Lasota et al. (1995). 
A criterion for the stability of (hot) irradiated disks in SXTs was derived by van Paradijs (1996) (see however Dubus et al. 1998). The criterion is shown as a dashed line in Fig. 9. All systems above this line are globally stable according to this criterion. Van Paradijs did not include the additional accretion occurring via the ADAF in quiescent BH SXTs. One might worry that including it may modify his results. However, as Fig. 9 shows, the accretion rate estimates $\left(\dot{M}_{\text {tot }}\right)$ for A0620-00, GS2000+25, Nova Mus 91 and Nova Oph 77, which take into account the additional accretion via the ADAF, are all below van Paradijs' stability line. The same is true for J0422+32, J1655-40 and V404 Cyg (not shown).

To summarize, the $P_{\text {orb }}-\dot{M}_{T}$ plane is divided into three zones. The region to the lower right in Fig. 9, below the solid line (which line we choose depends on the choice of $f_{t}$ ), corresponds to faint persistent systems in which the disk is in a globally stable cool state. No such systems have been found yet, but they may well exist and remain undetected, as they are likely to be very dim. The middle zone, between the solid and dashed lines corresponds to an unstable disk. Such systems are expected to be transients, and indeed all known dynamically confirmed BH SXTs are found in this region. Finally, the region above the dashed line corresponds to luminous persistent systems in which the irradiated disk is in a globally stable hot state. The persistent black hole binaries, Cyg X-1 and LMC X-3, both lie in this region. Neither is a LMBHB, but van Paradijs' criterion applies equally to high mass binaries (provided they accrete from a disk). A number of other persistent black hole binaries may also lie in this zone, but there is not yet enough dynamical information to determine their status.

Figure 9 shows that if transition radii in quiescent BH SXTs were slightly larger (i.e if $f_{t}$ were larger), some of the systems would become globally stable and would correspond to faint persistent LMBHBs. Do such systems exist in the Galaxy?

\section{A Galactic Population of Dim Accreting Binary BHs ?}

To answer the above question, we use models of binary evolution to predict the accretion rates in binary systems as a function of their orbital periods and check if any of the evolutionary tracks cross the lowermost zone in Fig. 9 .

\subsection{Mass Transfer from Binary Evolution Models}

The standard theory of binary evolution relies on the Roche-lobe model for the description of mass transfer in binary star systems. Depending on which mechanism drives the mass transfer, whether it is loss of orbital angular momentum through gravitational radiation and magnetic braking, or expansion of the donor as it evolves away from the main-sequence, the systems are classified as $\mathrm{j}$-driven or n-driven systems. There is a 'bifurcation' orbital period separating the two classes such that above this period we find n-driven systems whose $P_{\text {orb }}$ increase with time, and below this period we find $\mathrm{j}$-driven systems with $P_{\text {orb }}$ decreasing with time. The precise value of the bifurcation period depends on the history of the system. Estimates range from 0.5 - 2 days (e.g. Pylyser \& Savonije 1988, King, Kolb \& Burderi 1996).

Following King et al. (1996), we take the mass transfer rates in j-driven systems with gravitational radiation (GR) and magnetic braking (MB), and n-driven system with secondary expansion (ex), to be given by:

$$
\dot{M}_{\mathrm{GR}}=1.27 \times 10^{14} m_{1} m_{2}^{2}\left(m_{1}+m_{2}\right)^{-1 / 3} P_{\mathrm{d}}^{-8 / 3} \mathrm{~g} \mathrm{~s}^{-1},
$$




$$
\begin{gathered}
\dot{M}_{\mathrm{MB}}=3.17 \times 10^{17} m_{1}^{-1} m_{2}^{7 / 3}\left(m_{1}+m_{2}\right)^{1 / 3} P_{\mathrm{d}}^{-2 / 3} \mathrm{~g} \mathrm{~s}^{-1}, \\
\dot{M}_{\mathrm{ex}}=2.54 \times 10^{16} m_{2}^{1.47} P_{\mathrm{d}}^{0.93} \mathrm{~g} \mathrm{~s}^{-1} .
\end{gathered}
$$

Note that Eq. (21) is a large oversimplification; a substantial scatter around this prediction is expected, depending on the precise history of the system (see Fig. 10 for more accurate evolutionary tracks).

The exact form of the magnetic braking law is not well established. Eq. (20) corresponds to the formula due to Verbunt \& Zwaan (1981). Other models of magnetic braking have been proposed in the literature (see e.g. Warner 1995) but they give similar results (not differing by more than a factor of a few). Eq. (20) does not apply, however, to systems with orbital periods $\gtrsim 10 \mathrm{hr}$. The magnetic braking model represents well the secular mean accretion rate of cataclysmic variables and allows an understanding of the so called 'period-gap' in the orbital period distribution of cataclysmic binaries. (Contrary to some recent and less recent assertions, the period gap does exist, e.g. Hellier \& Naylor 1998.) One observes, however, a rather large scatter of mass transfer rates deduced from observations, a large part of which is clearly intrinsic (Warner 1995). This means that some systems and some classes of systems transfer mass at rates different from the secular one. These fluctuations are compatible with the secular evolution as long as the durations of the fluctuations are much shorter than the characteristic evolutionary time of the system $\left(\sim 10^{8}\right.$ years in the case of magnetic braking).

We plot in Fig 10a the predictions of the binary evolution model for GR driven (long-dashed line, Eq. (19)) and MB driven (dotted-dashed line, Eq. (20)) mass transfer rates, for orbital periods up to $P_{\text {orb }}=20 \mathrm{~h}$. At longer periods, we plot the predictions for secondary expansion corresponding to detailed evolutionary tracks rather than the crude Eq (21). The evolutionary tracks were computed with a semi-analytical model, described by Verbunt \& van den Heuvel (1995). It gives results which compare well with those of more detailed stellar evolution models since the properties of a (sub)giant star depend mainly on the mass of its core (Webbink, Rappaport \& Savonije 1983). Four examples of evolutionary tracks are shown in Fig 10a for binary systems with an initial mass of the secondary $m_{2}=1$ (dashed line) and 1.5 (dotted line), with two different initial periods (17 hr and $72 \mathrm{hr}$ ). The mass of the primary is $m_{1}=7$ in each case, but the predictions for the mass transfer rates increase by only $\sim 20 \%$ if the mass of the primary is $m_{1}=15$. The composition is assumed to be solar. Note that the specific evolutionary state of J1655-40 is probably not accurately represented by any of the evolutionary tracks shown (e.g. Kolb 1998).

Also plotted in Fig 10a are our estimates of $\dot{M}_{\text {tot }}$ for the seven known BH SXTs. While the predictions for GR driven and secondary expansion driven mass transfer (Eq. (19)) appear in reasonable agreement with the estimates of $\dot{M}_{\text {tot }}$, there is clearly a problem in the case of MB driven mass transfer (Eq. (20)), for which the theoretical predictios are well above the estimates of $\dot{M}_{\text {tot }}$ in J0422+32, A0620-00, GS2000+25, Nova Mus 91 and Nova Oph 77. One should keep in mind that the estimates of $\dot{M}_{\text {tot }}$ in GS2000+25 and Nova Mus 91 may well go down by as much as an order of magnitude (§3.1.3), which would make the discrepancy more serious. The disagreement would be even more acute if we did not invoke a low radiative efficiency ADAF in quiescence.

Quite apart from the inconsistency between the estimates of $\dot{M}_{\text {tot }}$ and the magnetic braking prediction, we note the serious problem that the MB line in Fig 10 a lies entirely above van Paradijs' stability criterion, shown by the solid line. If Eq. (20) is correct, then there should be no transient LMBHBs with $P_{\text {orb }}<20$ hr. But five of the seven transient systems we study have periods in this range. Equation (20) is clearly inapplicable to these systems.

How do we resolve this discrepancy? There is no theoretical reason or observational evidence to suggest that magnetic braking does not operate in LMBHBs. There are reasons, however, to think that the 
companions in LMBHBs could be evolved (King et al. 1996). In this case Eq. 20 becomes:

$$
\dot{M}_{\mathrm{MBev}}=3.17 \times 10^{17} m_{1}^{-1} \hat{m}_{2}^{7 / 3}\left(m_{1}+m_{2}\right)^{1 / 3} P_{\mathrm{d}}^{-2 / 3} \mathrm{~g} \mathrm{~s}^{-1},
$$

where $\hat{m}_{2}=m_{2} / m_{2, \text { main-sequence }} \approx 0.5-0.6$. A similar formula can be deduced for GR driven mass transfer with an evolved donor. The accretion rates predicted by "evolved" GR and MB laws are typically a factor 3 to 5 smaller than the predictions of Eq. (19) and Eq. (20). As suggested by King et al. (1996), this would bring the predictions closer to the observational estimates. The law of Verbunt \& Zwaan (1981) predicts relatively high accretion rates. Using a different law, for instance the Rappaport, Joss \& Verbunt (1983) law (see e.g. Kalogera, Kolb \& King 1998), would bring the predictions even closer to the observational estimates.

There is an additional argument, based on the recurrence times of BH SXTs, which shows that Eq. (20) is inappropriate to predict mass transfer rates in LMBHBs. This is discussed in the next subsection.

\subsection{Recurrence Times}

According to the DIM, the maximum surface density of a cold (quiescent) disk is (Hameury et al. 1998, see also e.g. Ludwig et al. 1994, Smak 1993):

$$
\Sigma_{\max }=13.1 \alpha^{-0.85} m_{1}{ }^{-0.37} R_{10}^{1.11} \mathrm{~g} \mathrm{~cm}^{-2} .
$$

The corresponding maximum quiescent disk mass is given by:

$$
M_{\max }=3.9 \times 10^{21} \alpha^{-0.85} m_{1}^{-0.37} R_{\mathrm{D}, 10}^{2.11} \mathrm{~g},
$$

where $R_{\mathrm{D}, 10}$ is the disk outer radius in units of $10^{10} \mathrm{~cm}$. As suggested by observations (e.g. Harrop-Allin \& Warner 1996) we will assume that $R_{\mathrm{D}}$ is about $70 \%$ of the Roche-lobe equivalent radius. Using an approximate formula by Paczyński (1971),

$$
R_{\mathrm{L}} \approx\left[0.38-0.2 \log \left(\frac{m_{2}}{m_{1}}\right)\right] a, \text { for } 0.3<\frac{m_{1}}{m_{2}}
$$

where $a$ is the binary separation, we obtain

$$
R_{\mathrm{D}, 10} \sim 15 m_{1}^{1 / 3} P_{\mathrm{orb}}^{2 / 3}
$$

with the orbital period $P_{\text {orb }}$ measured in days.

There is an important difference between WZ Sge-type systems and SXTs. During a (super)outburst of the short period dwarf nova WZ Sge, more than $10^{24} \mathrm{~g}$ of matter was accreted onto the central white dwarf (Smak 1993). Taking the disk radius in this system to be $\sim 10^{10} \mathrm{~cm}$, we see from Eq. (24) that $\alpha$ in quiescence must be very low $\left(\lesssim 10^{-4}\right)$, unless, due to irradiation of the secondary, mass transfer is increased during the outburst (Hameury, Lasota \& Huré 1997). The amount of mass accreted during SXT outbursts is also $\sim 10^{24} \mathrm{~g}$ but, since their orbital periods are between 0.2 and 6 days, the disks are large enough to contain the required mass even for a normal value of $\alpha$ (i.e. $\sim 10^{-2}$ ). Indeed, the secondary in WZ Sge showed evidence for irradiation during an outburst (Smak 1993) whereas there is no evidence for significant irradiation of the secondary in any SXT (McClintock, private communication). 
The recurrence time between two successive outbursts can be estimated very crudely as the time required to fill up the disk to its maximum mass:

$$
t_{\text {rec }} \lesssim \frac{M_{\max }}{\dot{M}_{\mathrm{T}}\left(1-\frac{\dot{M}_{\mathrm{acc}}}{\dot{M}_{\mathrm{T}}}\right)}
$$

where $\dot{M}_{\text {acc }}$ and $\dot{M}_{\mathrm{T}}$ are the accretion rate in quiescence at the inner disk radius (in the case of an ADAF + thin disk accretion flow, the 'inner' radius is the transition radius) and the mass transfer rate from the secondary, respectively. In the DIM framework, if the quiescent disk extends down to the last stable orbit (or to the surface of the compact body in the case of white dwarfs) $\dot{M}_{\mathrm{T}} \gg \dot{M}_{\text {acc }}$ (see Eq. (iD)) so that the recurrence time is simply given by $t_{\mathrm{rec}} \lesssim M_{\max } / \dot{M}_{\mathrm{T}}$. In the presence of an inner ADAF in quiescent SXT accretion flows (and in a similar model for WZ Sge by Hameury et al. 1997a), the 'leak' at the transition radius may require the use of Eq. (27). However, the term $\left(1-\dot{M}_{\text {acc }} / \dot{M}_{\mathrm{T}}\right)^{-1}$ is generally very close to unity because

$$
\frac{\dot{M}_{\mathrm{acc}}\left(0.2 \times R_{\mathrm{circ}}\right)}{\dot{M}_{\mathrm{T}}} \approx 0.04 m^{-1} m_{2}^{-7 / 3} m_{1}^{0.11} P_{\mathrm{d}}^{2.45} \alpha^{-0.04},
$$

where we use the critical accretion rate in Eq. (1) to estimate $\dot{M}_{\text {acc }}$.

In order to estimate recurrence times in the ADAF + thin disk model, we use the mass-transfer rates given in Eqs. (19)-(21). Combining these with Eqs. (24) and (26), we find

$$
\begin{aligned}
\mathrm{GR}: t_{\mathrm{rec}} & =295 \mathrm{y} \alpha^{-0.85} m_{1}^{-0.67} m_{2}^{-2}\left(m_{1}+m_{2}\right)^{1 / 3} P_{\mathrm{d}}^{4.08}\left(1-\frac{\dot{M}_{\mathrm{acc}}}{\dot{M}_{\mathrm{T}}}\right)^{-1}, \\
\mathrm{MB}: t_{\mathrm{rec}} & =0.3 \mathrm{y} \alpha^{-0.85} m_{1}^{1.33} m_{2}^{-7 / 3}\left(m_{1}+m_{2}\right)^{-1 / 3} P_{\mathrm{d}}^{2.08}\left(1-\frac{\dot{M}_{\mathrm{acc}}}{\dot{M}_{\mathrm{T}}}\right)^{-1} \\
\text { ex }: t_{\mathrm{rec}} & =1.47 \mathrm{y} \alpha^{-0.85} m_{1}^{0.33} m_{2}^{-1.47} P_{\mathrm{d}}^{0.48}\left(1-\frac{\dot{M}_{\mathrm{acc}}}{\dot{M}_{\mathrm{T}}}\right)^{-1} .
\end{aligned}
$$

Using mass-period relations for main sequence stars $\left(m_{2} \approx 0.11 P_{\mathrm{h}}\right)$ and for subgiants $\left(m_{2} \propto P^{-0.33}\right.$, e.g. King 1988) we obtain acceptable estimates of the recurrence time for the cases of gravitational radiation and secondary evolution. The recurrence time for the magnetic braking case is, however, far too short. This has also been noticed by Romani (1998). The use of Eq. (22), instead of Eq. (20), makes the estimated recurrence time longer if we take $\hat{m}_{2} \approx 0.5-0.6$.

There is a clear need for better predictions of the mass transfer rates via magnetic braking in low orbital period systems. Direct observational constraints on magnetic braking in cataclysmic variables are scarce (Kolb, private communication) and the extrapolation of these data to LMBHBs is hazardous. For these reasons, we choose to ignore the contribution of magnetic braking to the mass transfer rate in what follows.

\subsection{Do Faint Persistent LMBHBs exist?}

Figure 10b shows the same predicted mass transfer rates as in Fig. 10a, for GR driven and secondary expansion driven mass transfer, but omitting the problematic MB case. Three stability criteria (solid lines, 
$f_{t}=0.3,0.2,0.1$ from above) are also shown, for a 'typical' system with $m_{1}=7$ and $m_{2}=\min \left[0.11 P_{\mathrm{hr}}, 1\right]$. Recall that a system that is below one of these lines will be faint and persistent.

At short orbital periods ( $P_{\text {orb }} \lesssim 20 \mathrm{~h}$ ), binary evolution models predict accretion rates which are substantially above the limit for stability. We do not expect faint persistent LMBHBs in this range of periods for any reasonable value of $f_{t}$. This conclusion is independent of the uncertainty on the magnetic braking law because magnetic braking only predicts larger accretion rates.

At long periods, however, some of the evolutionary tracks do predict accretion rates low enough to allow the existence of faint LMBHBs. This is especially true for $f_{t} \sim 0.2-0.3$, but some persistent systems are expected even if $f_{t} \sim 0.1$. Thus, faint persistent LMBHBs are viable at long orbital periods, provided that transition radii in these systems are not too small (i.e $f_{t}$ not $\ll 0.1$ ). What would such systems look like?

\subsection{Observational Signatures of Faint Persistent LMBHBs}

The main difference between a faint persistent LMBHB and a quiescent SXT is that $r_{\mathrm{tr}}>r_{\text {crit }}$ in the former and $r_{\mathrm{tr}}<r_{\text {crit }}$ in the latter. Of course, since $r_{\text {crit }}$ is a function of $\dot{m}$, variations of the mass-transfer rate can move systems from one class to another.

In both kinds of system, $r_{\text {tr }}$ is sufficiently large that the emission from the outer thin disk is weak and unimportant relative to the emission from the secondary star. Further, the emission from the ADAF is dominated by the regions of the flow close to the black hole $(r<30)$, which means that the precise value of $r_{\mathrm{tr}}$ is unimportant. For both reasons, we expect the spectrum of a faint persistent LMBHB to be very similar to that of a quiescent SXT with the same mass accretion rate (or similar orbital period).

By the arguments given earlier, we expect faint persistent LMBHBs to have relatively long orbital periods $\left(P_{\text {orb }}>30 \mathrm{hr}\right)$ and fairly large mass transfer rates $\left(10^{17} \mathrm{~g} \mathrm{~s}^{-1}>\dot{M}_{T}>10^{16} \mathrm{~g} \mathrm{~s}^{-1}\right)$. We show in Fig 11 predicted spectra of representative faint persistent LMBHBs (solid lines) as they would appear if the sources were located at a distance of $1 \mathrm{kpc}$. Three different accretion rates are shown, corresponding to a black hole mass $m_{1}=7$ and $\alpha_{\mathrm{ADAF}}=0.3$. A comparison between Fig. 11 and Figs. 7 and 8 reveals the strong spectral similarity between faint persistent LMBHBs and quiescent SXTs.

In the three known BH SXTs with long orbital periods, namely V404 Cyg $\left(P_{\text {orb }}=155.4 \mathrm{hr}\right), \mathrm{J} 1655-40$ $(62.7 \mathrm{hr})$ and $4 \mathrm{U} 1543-47(26.9 \mathrm{hr})$, the secondary dominates the optical and IR emission in quiescence (this is true even in the case of the cool companion in V404 Cyg where the disk contributes less than about $15 \%$ in optical and IR; Casares et al. 1993; Shahbaz et al. 1996). We expect faint persistent LMBHBs to have subgiant secondaries similar to those found in these three BH SXTs. We show in Fig 11 model black body spectra corresponding to the secondaries in V404 Cyg, GRO J1655-40 and 4U 1543-47. As expected, the secondaries dominate the emission in the optical band; the accretion flow contributes very little. Clearly, it would be very difficult to discover faint persistent LMBHBs via any kind of broadband survey in the optical. One could in principle look for excess emission in blue and ultraviolet radiation, arising from synchrotron radiation in the ADAF, but this is not likely to be a very effective technique.

Of course, if any particular system is suspected to be a faint persistent LMBHB, it would be relatively easy to verify its LMBHB nature through observations. The orbital motion of the secondary could be confirmed through measurements of the Doppler shifts of its spectral lines. This would reveal the mass function of the binary and may even lead to a determination of the mass of the black hole (as in the best 
BH SXTs). Also, the detection of a broad double-humped $H_{\alpha}$ line would reveal the presence of the outer thin accretion disk, just as in quiescent BH SXTs. Finally, observations in X-rays would reveal the presence of the ADAF.

Another severe problem is that even if one did discover a candidate system, it will not be easy to confirm that it is a faint persistent LMBHB rather than a quiescent SXT. SXTs have typical recurrence times of 20 to 50 years. One would have to observe the candidate system for at least 50 years before one could be reasonably confident that it is not a SXT.

\section{Discussion}

Whether or not faint persistent LMBHBs exist in the Galaxy depends on the mass transfer rates $\dot{M}_{T}$ and transition radii $R_{\mathrm{tr}}$ in black hole binaries. While our predictions of the mass transfer rates, especially via secondary expansion, are relatively robust, this is not the case for our estimates of $R_{\mathrm{tr}}$. We present a specific prescription for estimating the transition radius, based on stream dynamics and other considerations. Observations of $H_{\alpha}$ emission lines provide only upper limits to the values of $R_{\mathrm{tr}}$. In the absence of a more complete theory of gas evaporation from the thin disk to the ADAF, the location of the transition radius remains a major uncertainty of the model. Indeed, until this issue is resolved it is difficult to estimate what fraction of the LMBHB population in the Galaxy might correspond to faint persistent systems.

Another uncertainty is related to time variability of the mass transfer rate. We have shown that the known SXTs have outer disks that are unstable to the thermal-viscous instability of the DIM, and therefore that these systems would be unstable even with a steady mass transfer from the secondary. However, Kuulkers et al. (1996) recently emphasized the striking similarities between the lightcurves of WZ Sge type dwarf novae and SXTs. In the case of the WZ Sge type systems, one needs to invoke a variable mass transfer rate, with an enhancement during the outburst, if one wishes to avoid using extremely low values of the viscosity parameter $\alpha$ (see Hameury et al. 1997a). If LMBHBs have similar variable mass transfer rates, then even those systems that ought to be faint and persistent by virtue of their mean $\dot{M}_{T}$ could be driven into transient behavior by fluctuations in $\dot{M}_{T}$. Note also that the existence of faint persistent LMBHBs is unlikely if the transition radii are substantially smaller than the observational upper limits inferred in quiescent BH SXTs, or if $\alpha_{\mathrm{ADAF}}$ is smaller than 0.3 (e.g. 0.1).

Currently, there is a discrepancy between theoretical estimates of the rate of formation of black hole binaries and observational estimates of the number of LMBHBs in the Galaxy; the former falls short by a significant factor (e.g. Portegies Zwart, Verbunt \& Ergma 1997). If in addition to the known population of SXTs there were also an undiscovered population of faint persistent LMBHBs, the discrepancy would clearly increase.

We have not considered Low Mass Neutron Star Binaries for several reasons. First, the energy advected via the ADAF onto a NS would be reradiated from the NS surface (Narayan, Garcia \& McClintock 1997); the reemitted radiation would modify the structure of the ADAF (Narayan \& Yi, 1995) and, to some extent, the stability properties of the thin disk as well (van Paradijs 1996). Second, given the uncertainties in the structure and emission spectrum of the boundary layer between the accretion flow and the NS surface, it is difficult to make any quantitative predictions. Finally, LMNSBs may exhibit a strong 'propeller' effect (e.g. Zhang, Yu \& Zhang 1998) which is difficult to model. 
On the other hand, work on faint persistent LMNSBs is well worth the effort. Because the advected energy is reradiated from the NS surface, faint persistent LMNSBs are expected to be brighter than faint persistent LMBHBs. A population of faint persistent LMNSBs may thus be easier to identify than their black hole counterparts. Recurrence time scales in LMNSBs are also shorter than in LMBHBs. Therefore, one could more quickly determine whether a candidate LMNSB is persistent or transient.

\section{Conclusion}

In this paper, we have investigated the global stability of LMBHBs assuming that the accretion flow around the black hole is composed of two zones: an ADAF for radii less than a transition radius $R_{\mathrm{tr}}$ and a thin accretion disk for radii beyond $R_{\mathrm{tr}}$.

The critical issue is the location of the transition radius. Observations of $H_{\alpha}$ emission line widths constrain $R_{\mathrm{tr}}$ in quiescent BH SXTs (§2.1.2). The observational constraints are consistent with an empirical prescription we have developed for predicting $R_{\mathrm{tr}}$ on the basis of stream dynamics (§2.1.1) and ADAF physics $(\S 2.1 .3)$.

The second issue is the mass transfer rates $\dot{M}_{T}$ in LMBHBs. We estimate $\dot{M}_{T}$ in the known SXTs from observations ( $\$ 3.1 .1$ and 3.1.2) and compare the estimates with the rates predicted by theoretical models of binary evolution under the influence of gravitational radiation, magnetic braking and secondary expansion (§4.1). The observational and theoretical estimates are in general agreement, except in the case of magnetic braking where the predicted mass transfer rate is much too large. For this and other reasons (explained in $\S 4.1$ and $\S 4.2$ ) we assume that the current prescriptions for magnetic braking are invalid in BH SXTs and ignore magnetic braking in our models.

By combining the theoretical and observational input on $R_{\mathrm{tr}}$ and $\dot{M}_{T}$, and comparing the derived values with the standard model of the thermal-viscous instability in disks, we show that all the known LMBHBs should be transients. This is consistent with the fact that they are all known to be SXTs, providing general confirmation of our methods.

Our model also predicts that a number of LMBHBs, especially those with somewhat longer orbital periods, might be non-transients. These steady systems would be faint and persistent, and extremely difficult to discover. The prediction of such a population of black hole binaries is the main result of the paper. We calculate model spectra of these systems, and show that the spectra are very similar to those of quiescent SXTs. Unfortunately, we have been unable to come up with an efficient search strategy to find these faint persistent LMBHBs. We are also unable to estimate how large a population these systems may constitute relative to the known population of BH SXTs.

\section{Acknowledgments}

We are grateful to Vicky Kalogera, Uli Kolb and Jeff McClintock for useful discussions and we thank Phil Charles, Jean-Marie Hameury and Eliot Quataert for comments on the manuscript. Insu Yi provided the cooling function in Fig. 2a. This work was supported in part by NASA grant NAG 5-2837. KM was supported by a SAO Predoctoral Fellowship and a French Higher Education Ministry Grant. 


\section{REFERENCES}

Abramowicz, M.A.,Chen, X.-M., Kato, S., Lasota, J.-P. \& Regev, O., 1995, ApJ Lett. 438, L37.

Abramowicz, M.A., Igumenshchev, I.V. \& Lasota, J.-P., 1998, MNRAS 293, 443.

Barret, D., McClintock, J.E., \& Grindlay, J.E., 1996, ApJ 473, 963.

Beekman, G., et al., 1997, MNRAS 290, 303.

Callanan, P.J. et al., 1996, ApJ 461, 351.

Cannizzo, J.K., 1993, in Accretion Disks in Compact Stellar Systems, ed J.C. Wheeler, (Singapore: World Scientific), p. 6.

Cannizzo, J.K., Ghosh, P. \& Wheeler, J.C., 1985, ApJ Lett. 260, L83.

Cardelli, J.A., Clayton, G.C. \& Mathis, J.S. 1989, ApJ 345, 245.

Charles, P.A., Kidger, M.R., Pavlenko, E.P., Prokof'eva, V.V., \& Callanan, P.J., 1991, MNRAS $249,567$.

Casares, J., Charles, P.A., Naylor, T. \& Pavleno E.P., 1993, MNRAS 265, 834.

Chen, X.M., Abramowicz, M.A. \& Lasota, J.-P., 1997, ApJ 476, 61.

Chen, X.M., Abramowicz, M.A., Lasota, J.-P., Narayan, R. \& Yi. I., 1995, ApJ Lett. 443, L61.

Chen, W., Shrader, C.R., \& Livio, M., 1997, ApJ 491, 312.

Dalgarno, A. \& McCray, R.A., 1972, ARAA 10, 375.

Dubus G., Lasota, J.-P., Hameury, J.-M. \& Charles, P., 1998, MNRAS submitted.

Esin, A.A., 1997, ApJ 482, 400.

Esin, A.A., McClintock, J.E., \& Narayan, R., 1997, ApJ 489, 865.

Esin, A.A., Narayan, R., Cui, W., Grove, J.E. \& Zhang, S.N., 1998, ApJ in press, Astro-ph/9711167.

Filippenko, A.V., Matheson, T., \& Barth, A.J., 1995, ApJ 455, 614.

Filippenko, A.V., Matheson, T., Leonard, D.C., Barth, A.J., \& Van Dyk, S.D., 1997, PASJ $109,461$.

Frank, J., King, A. \& Lasota, J.-P., 1987, A\&A 178, 137.

Frank, J., King, A. \& Raine, D., 1992, Accretion Power in Astrophysics (Cambridge: Cambridge University Press).

Gammie, C.F., Blandford, R. \& Narayan, R., 1998, in preparation.

Garcia, M.R., Callanan, P.J., McClintock, J.E., \& Zhao, P., 1996, ApJ 460, 932.

Garcia, M.R., McClintock, J.E., Narayan, R., \& Callanan, J., 1997, in the Proceedings of the 13th NAW on CVs, Jackson Hole, WY, eds. S. Howell, E. Kuulkers, and C. Woodward, Astro-ph/9708149.

Griffiths, R.E., et al., 1978, ApJ Lett. 221, L63. 
Hameury, J.-M., Lasota, J.-P. \& Huré, J.-M., 1997a, MNRAS 287, 937.

Hameury, J.-M., Lasota, J.-P., McClintock, J.E., \& Narayan, R., 1997b, ApJ 489, 234.

Hameury, J.-M., Menou, K., Dubus, G., Lasota, J.-P. \& Huré, J.-M. 1998, MNRAS, in press, Astro$\mathrm{ph} / 9803242$.

Harlaftis, E.T., Horne, K., \& Filippenko, A.V., 1996, PASJ 108, 762.

Harlaftis, E.T., Steeghs, D., Horne, K., \& Filippenko, A.V., 1997, Astron. J. 114, 1170.

Harrop-Allin, M.K. \& Warner, B., 1996, MNRAS 279, 219.

Hellier, C. \& Naylor, T., 1998, MNRAS in press, Astro-ph/9802041.

Hjellming, R.M. \& Rupen, M.P., 1995, Nature 375, 464.

Honma, F., 1996, PASJ 48, 77.

Huang, M. \& Wheeler, J.C., 1989, ApJ 343, 229

Hynes, R.I. et al., MNRAS in press, Astro-ph/9803145.

Kalogera, V., Kolb, U. \& King, A., 1998, ApJ in press, Astro-ph/9803288.

Kato, S., Abramowicz, M.A. \& Chen, X.-M., 1996, PASJ 48, 67.

Kato, S., Yamasaki, T., Abramowicz, M.A. \& Chen, X.M. 1997, PASJ 49, 221.

King, A., 1988, Quat. J. R. Astron. Soc. 29, 1.

King, A., 1993, MNRAS 260, L5.

King, A. \& Ritter, H., 1998, MNRAS 293, 42

King, A., Kolb, U. \& Burderi, L., 1996, ApJ Lett. 464, L127.

King, A., Kolb, U. \& Szuszkiewicz, E., 1997, ApJ 488, 89.

Kolb, U., 1998, MNRAS in press, Astro-ph/9801283.

Kuulkers, E., Howell, S. B. \& van Paradijs, J., 1996, ApJ Lett. 462, L87.

Lasota, J.-P., 1996a, in Compact Stars in Binaries; IAU Symposium 165, eds. J. van Paradijs E.P.J. van den Heuvel \& E. Kuulkers, (Dordrecht: Kluwer), p. 43.

Lasota, J.-P., 1996b, in Cataclysmic Variables and Related Objects, IAU Coll. 158, eds. A. Evans \& J.H. Wood (Dordrecht: Kluwer) p. 385.

Lasota, J.-P., 1998, in Astrophysical Fluids: From Atomic Nuclei to Stars and Galaxies, Physics Reports, in press, Astro-ph/9806064.

Lasota, J.-P. \& Hameury, J.-M., 1998, in Accretion Processes in Astrophysics - Some Like it Hot, eds. S. Holt \& T. Kallman, in press, Astro-ph/9712202.

Lasota, J.-P. \& Hameury, J.-M. \& Huré, J.-M., 1995, A\&A 302, 29. 
Lasota, J.-P., Narayan, R. \& Yi, I., 1996, A\&A 314, 813.

Lubow, S.H., 1989, ApJ 340, 1064.

Lubow, S.H. \& Shu, F.H., 1976, ApJ Lett. 207, L53.

Ludwig, K., Meyer-Hofmeister, E. \& Ritter, H., 1994, A\&A 290, 473.

Mahadevan, R. \& Quataert, E., 1997, ApJ 490, 605.

Meyer, F. \& Meyer-Hofmeister, E., 1994, A\&A 288, 175.

McClintock, J.E., Petro, L.D., Remillard, R.A. \& Ricker, G.R., 1983, ApJ Lett. 266, L27.

Meyer, F. \& Meyer-Hofmeister, E., 1994, A\&A 288, 175.

Mineshige, S. \& Wheeler, J.C., 1989, ApJ 343, 241.

Nakamura, K.E., Kusunose, M., Matsumoto, R. \& Kato, S., 1997, PASJ 49, 503.

Narayan, R., Barret, D., \& McClintock, J.E., 1997a, ApJ 482, 448.

Narayan, R., Garcia, M.R. \& McClintock, J.E., 1997c, ApJ Lett. 478, L79.

Narayan, R., Kato, S. \& Honma, 1997b, ApJ 476, 49.

Narayan, R., Mahadevan, R., Grindlay, J.E., Popham, R.G. \& Gammie, C.F., 1998, ApJ 492, 554.

Narayan, R., Mahadevan, R. \& Quataert, E., 1998, in The Theory of Black Hole Accretion Discs, eds. M. A. Abramowicz, G. Bjornsson, and J. E. Pringle (Cambridge: Cambridge University Press), Astro-ph/9803141.

Narayan, R., McClintock, J.E. \& Yi, I., 1996, ApJ 457, 821.

Narayan, R. \& Yi, I., 1994, ApJ Lett. 428, L13.

Narayan, R. \& Yi, I., 1995, ApJ 444, 231.

Orosz, J.A., \& Bailyn, C.D., 1995, ApJ Lett. 446, L59.

Orosz, J.A., \& Bailyn, C.D., 1997, ApJ 477, 876.

Orosz, J.A., Jain, R.K., Bailyn, C.D., McClintock, J.E. \& Remillard, R.A., 1998, ApJ 499, 375.

Paczyński, B., 1971, ARAA 9, 189.

Patterson, J., Richman, H., Kemp, J. \& Mukai, K., 1997, PASP 110403

Piran, T., 1978, ApJ 221, 652.

Popham, R.G. \& Gammie, C.F., ApJ in press, Astro-ph/9802321.

Portegies Zwart, S.F., Verbunt, F. \& Ergma, E., 1997, A\&A 321, 207.

Pylyser, E. \& Savonije, G.J., 1988, A\&A 191, 57.

Rappaport, S., Joss, P.C. \& Verbunt, F., 1983, ApJ 275, 713. 
Romani, R.W., 1998, A\&A 333, 583.

Shahbaz, T., Bandyopadhyay, R., Charles, P.A. \& Naylor, T., 1996, MNRAS 282, 977.

Shakura, N.I \& Sunyaev, R.A., 1973, A\&A 24, 337.

Shapiro, S.L., Lightman, A.P. \& Eardley, D.M, 1976, ApJ 204, 187.

Smak, J.I., 1993, Acta Astronomica 43, 101.

Tanaka, Y. \& Lewin W.H.G., 1995, in X-ray Binaries, eds. W.H.G. Lewin, J. van Paradijs \& E.P.J. van den Heuvel, (Cambridge: Cambridge University Press), p. 126.

Tanaka, Y. \& Shibazaki, N., 1996, ARAA 34, 607.

Uno, S., Mitsuda, K., Aoki, T. \& Makino, F., 1997, PASJ 49, 353.

van Paradijs, J., 1996, ApJ Lett. 464, L139.

van Paradijs, J. \& McClintock, J.E., 1995, in X-ray Binaries, eds. W.H.G. Lewin, J. van Paradijs \& E.P.J. van den Heuvel, (Cambridge: Cambridge University Press), p. 58.

van Paradijs, J. \& Verbunt, F., 1984, in High Energy Transients in Astrophysics, AIP Conf., p. 49.

Verbunt, F. \& van den Heuvel, E.P.J., 1995, in X-ray Binaries, eds. W.H.G. Lewin, J. van Paradijs \& E.P.J. van den Heuvel, (Cambridge: Cambridge University Press), p. 457.

Verbunt, F. \& Zwaan, C., 1981, A\&A 100, L7.

Warner, B., 1995, Cataclysmic Variable Stars, (Cambridge: Cambridge University Press).

Webbink, R.F., Rappaport, S. \& Savonije, G.J., 1983, ApJ 270, 678.

Wheeler, J.C., 1996, in Relativistic Astrophysics, eds. B. Jones \& D. Markovic (Cambridge: Cambridge University Press).

White, N.E., Nagase, F. \& Parmar, A.N., 1995, in X-ray Binaries, eds. W.H.G. Lewin, J. van Paradijs \& E.P.J. van den Heuvel, (Cambridge: Cambridge University Press), p. 1.

Wu, X., 1997, MNRAS 292, 113.

Zhang, S.N., Cui, W. \& Chen, W., 1997, ApJ Lett. 482, L155.

Zhang, S.N., Yu, W. \& Zhang, W., 1998, ApJ Lett. 494, L71.

Życki, P.T., Done, C. \& Smith, D.A., 1998, in Accretion Processes in Astrophysics - Some Like it Hot, eds. S. Holt \& T. Kallman, in press, Astro-ph/9712190. 
Table 1: BH SXTs AND OUTBURST PARAMETERS

\begin{tabular}{|c|c|c|c|c|c|c|c|}
\hline $\begin{array}{l}\text { System } \\
\text { (1) }\end{array}$ & $\begin{array}{r}P_{\text {orb }}(\mathrm{hr}) \\
(2)\end{array}$ & $\begin{array}{c}\mathrm{D}(\mathrm{kpc}) \\
(3)\end{array}$ & $\begin{array}{r}\mathrm{i}\left({ }^{\circ}\right) \\
(4)\end{array}$ & $\begin{array}{c}m_{1}\left(M_{\odot}\right) \\
(5)\end{array}$ & $\begin{array}{c}m_{2}\left(M_{\odot}\right) \\
(6)\end{array}$ & $\begin{array}{c}\Delta F\left(\operatorname{ergs~} \mathrm{cm}^{-2}\right) \\
(7)\end{array}$ & $\begin{array}{c}\Delta t(\mathrm{yr}) \\
\quad(8)\end{array}$ \\
\hline GRO J0422+32 (XN Per. 92) & 5.1 & $2.6^{a}$ & $30^{g}$ & $12^{g}$ & $0.4^{e}$ & $1.2 \times 10^{-2}$ & $>30$ \\
\hline A0620-00 (XN Mon. 75) & 7.8 & $1^{b}$ & $55^{b}$ & $6.1^{b}$ & $0.55^{e}$ & 3.3 & 58 \\
\hline GS2000+25 (XN Vul. 88) & 8.3 & $2.7^{b}$ & $65^{e}$ & $8.5^{e}$ & $0.6^{e}$ & 2.2 & $>30$ \\
\hline GS1124-683 (XN Mus. 91) & 10.4 & $5^{c}$ & $60^{c}$ & $6^{c}$ & $0.8^{e}$ & 0.5 & $>30$ \\
\hline H1705-250 (XN Oph. 77) & 16.8 & $8.6^{b}$ & $70^{e}$ & $4.9^{e}$ & $0.7^{e}$ & 0.14 & $>30$ \\
\hline GRO J1655-40 (XN Sco. 94) & 62.7 & $3.2^{d}$ & $70^{d}$ & $7^{d}$ & $2.3^{f}$ & $4 \times 10^{-2}$ & $>30$ \\
\hline GS2023+338 (V404 Cyg) & 155.4 & $3.5^{b}$ & $56^{b}$ & $12^{b}$ & $0.9^{e}$ & 3.7 & 33 \\
\hline
\end{tabular}

NOTE. - (1) All dynamically confirmed BH SXTs are listed here with the exception of 4U 1543-47 which does not have adequate data. (2) Orbital periods from van Paradijs (1996). (3) Distances to the systems. (4) Inclinations. (5) Black hole masses. (6) Companion masses. (7) Outburst fluences from Chen et al. (1997), taking into account the listed distances. (8) Inter-outburst timescales, assuming that no outburst was missed (see Chen et al. (1997) for the reliability of the assumption that the coverage of the X-ray sky was complete during the last 30 years). (a) McClintock, private communication (1997). (b) Narayan et al. (1997c). (c) Esin et al. (1997). (d) Hameury et al. (1997b). (e) Chen et al. (1997). (f) Orosz \& Bailyn (1997). (g) Beekman et al. (1997). 
Table 2: OBSERVATIONAL DATA USED FOR THE ADAF MODELS OF GRO J0422+32, GS2000+25 AND NOVA OPH. 77

\begin{tabular}{lccccc}
\hline \hline $\begin{array}{l}\text { System } \\
(1)\end{array}$ & $\begin{array}{c}\lambda_{\text {opt }}(\AA) \\
(2)\end{array}$ & $\begin{array}{c}A_{V}(\mathrm{mag}) \\
(3)\end{array}$ & $\begin{array}{c}\% \text { from "disk" } \\
(4)\end{array}$ & $\begin{array}{c}\text { optical } \nu F_{\nu} \\
(5)\end{array}$ & $\begin{array}{c}\text { X-ray } \nu F_{\nu} \\
(6)\end{array}$ \\
\hline GRO J0422+32 (XN Per. 92) & 6300 & 1.2 & $30-60$ & $3.66_{-2.2}^{+3.16} \times 10^{-14}$ & $<2.7 \times 10^{-14}$ \\
GS2000+25 (XN Vul. 88) & 6250 & 5 & $1-11$ & $1.87_{-1.59}^{+1.85} \times 10^{-13}$ & $<1.2 \times 10^{-13}$ \\
H1705-250 (XN Oph. 77) & 6250 & 0.5 & $63-72$ & $8.1_{-3.41}^{+6.9} \times 10^{-14}$ & $<3 \times 10^{-13}$ \\
\hline
\end{tabular}

NOTE. - (2) Optical wavelength of observation. (3) Extinctions to the systems in the V band, from Callanan et al. (1996), Charles et al. (1991), Griffiths et al. (1978). (4) Fraction of light at $\lambda_{\text {opt }}$ that comes from the accretion flow, from Filippenko et al. (1995), Harlaftis et al. (1996), Harlaftis et al. (1997). (5) Net fluxes in $\operatorname{ergs~s}^{-1} \mathrm{~cm}^{-2}$ at the wavelength $\lambda_{\text {opt }}$. The original fluxes are taken from Filippenko et al. (1995), Harlaftis et al. (1996), Filippenko et al. (1997)) and have been corrected for interstellar extinction and contamination by the light from the secondary. (6) X-ray upper limits, in $\mathrm{ergs} \mathrm{s}^{-1} \mathrm{~cm}^{-2}$, from Garcia et al. (1997). 
Table 3: ACCRETION RATE ESTIMATES

\begin{tabular}{lccc}
\hline \hline & $\dot{M}_{\text {accum }}\left(\mathrm{g} \mathrm{s}^{-1}\right)$ & $\dot{M}_{\text {ADAF }}\left(\mathrm{g} \mathrm{s}^{-1}\right)$ & $\dot{M}_{\text {tot }}\left(\mathrm{g} \mathrm{s}^{-1}\right)$ \\
$\begin{array}{l}\text { System } \\
\text { 1) }\end{array}$ & $(2)$ & $(3)$ & $(4)$ \\
\hline GRO J0422+32 (XN Per. 92) & $<8.3 \times 10^{14}$ & $1.5-2.2 \times 10^{15}$ & $1.5-4.5 \times 10^{15}$ \\
A0620-00 (XN Mon. 75) & $2.5 \times 10^{15}$ & $2.1-3.3 \times 10^{15}$ & $3-8.7 \times 10^{15}$ \\
GS2000+25 (XN Vul. 88) & $<1.2 \times 10^{16}$ & $1.2-2.6 \times 10^{15}$ & $<0.9-2.2 \times 10^{16}$ \\
GS1124-683 (XN Mus. 91) & $<2.1 \times 10^{16}$ & $1.9 \times 10^{15}$ & $<1.5-3.6 \times 10^{16}$ \\
H1705-250 (XN Oph. 77) & $<3.5 \times 10^{15}$ & $2.7-6.8 \times 10^{15}$ & $4.1-15.5 \times 10^{15}$ \\
GRO J1655-40 (XN Sco. 94) & $<5.9 \times 10^{14}$ & $1.3-2.9 \times 10^{16}$ & $0.9-4.4 \times 10^{16}$ \\
GS2023+338 (V404 Cyg) & $5.9 \times 10^{16}$ & $2.2-3 \times 10^{16}$ & $5.4-13.3 \times 10^{16}$ \\
\hline
\end{tabular}

NOTE. - (2) Assumes a radiative efficiency $\eta=0.1$ for accretion in outburst. (3) From the ADAF spectral models shown in Figs. 7 and 8. (4) Sum of the two previous columns, with an additional uncertainty of a factor of 1.5 on the upper and lower limits. 


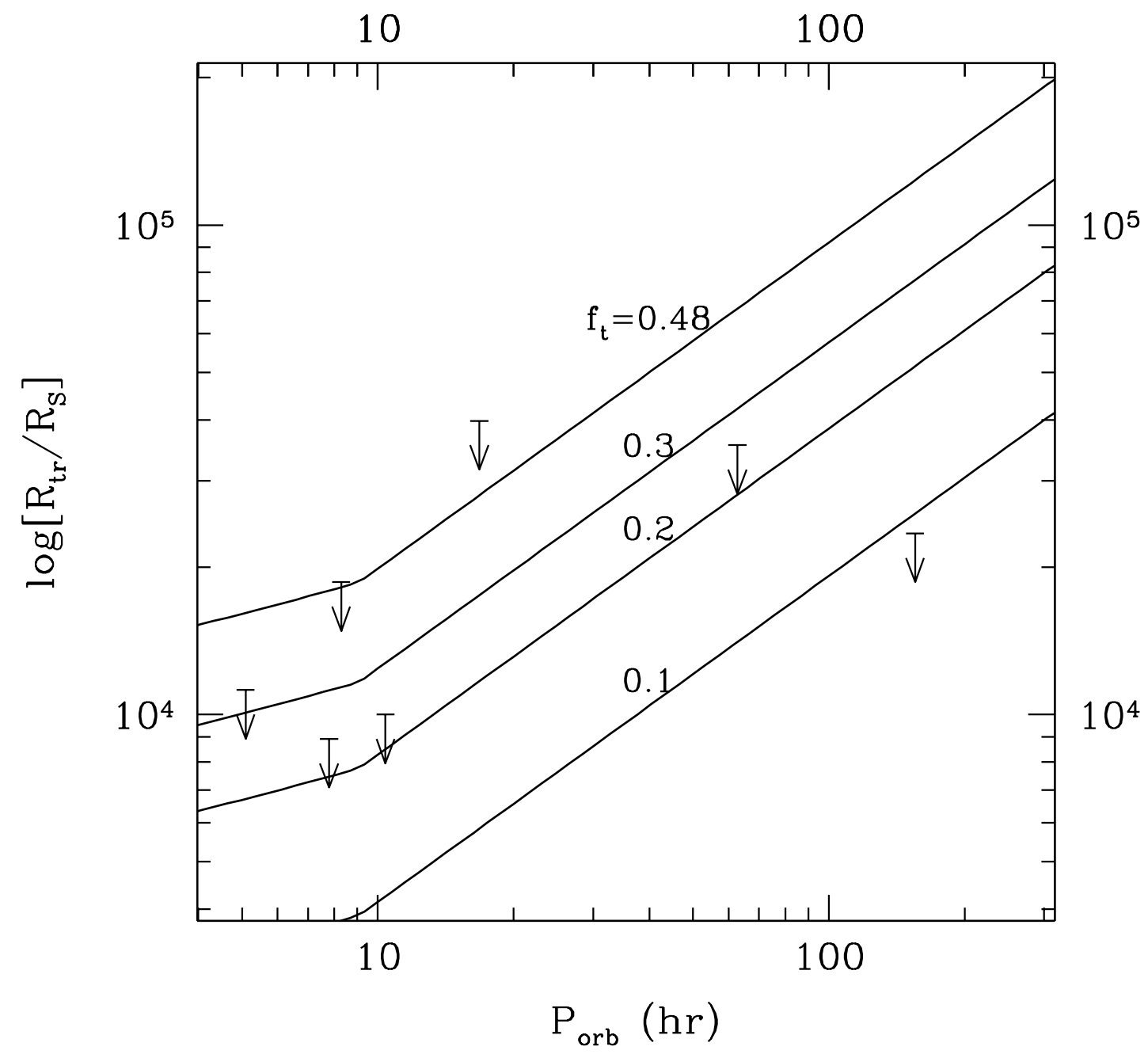

Fig. 1. - Shows observational upper limits (downward arrows) on the transition radius $R_{\mathrm{tr}}$ in the seven quiescent BH SXTs listed in Table 1. The limits were obtained from the widths of the $H_{\alpha}$ emission line in the quiescent spectra of the systems. Solid lines show the predicted variation of $R_{\mathrm{tr}}$ with orbital period, based on stream dynamics, for a 'typical' LMBHB with $m_{1}=7$ and $m_{2}=\min \left[0.11 P_{\mathrm{hr}}, 1\right]$. Four cases are shown, with $f_{t} \equiv R_{\mathrm{tr}} / R_{\text {circ }}=0.48,0.3,0.2,0.1$. The $H_{\alpha}$ line limits require that $f_{t} \lesssim 0.25$, except in the long period system V404 Cyg which requires $f_{t} \lesssim 0.1$. 

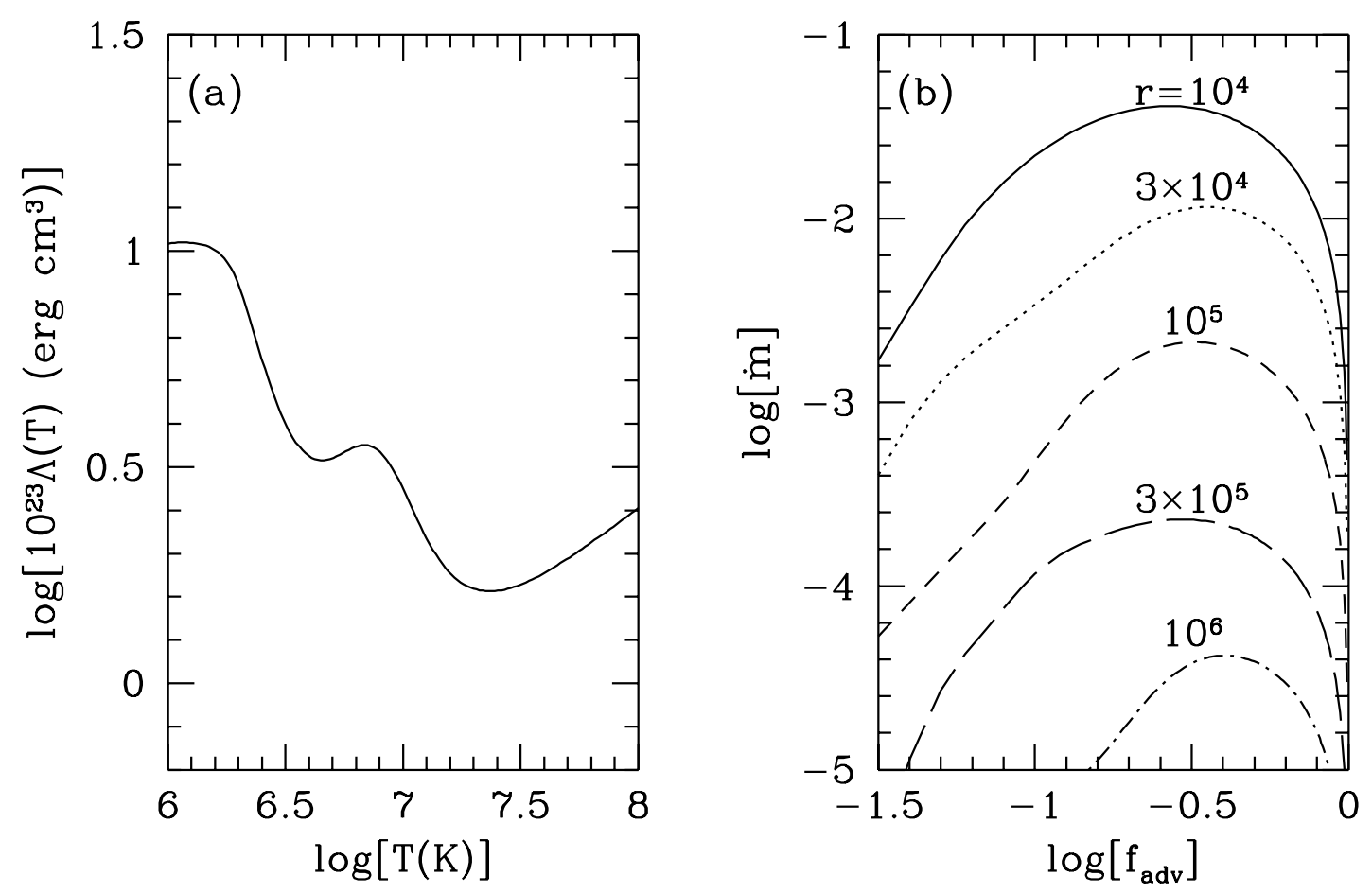

Fig. 2.- (a) Shows the cooling function $\Lambda(T)$ used in the calculations. (b) Shows the mapping between the dimensionless mass accretion rate $\dot{m}$ in an ADAF and the advection parameter $f_{\text {adv }}$, at five dimensionless radii $r$. The model assumes $\alpha_{\mathrm{ADAF}}=0.3$ and $P_{\text {gas }}=P_{\mathrm{mag}}$, or $\beta=0.5$ (i.e. exact equipartition). The solid, dotted, dashed, long-dashed and dotted-dashed lines correspond to $r=10^{4}, 3 \times 10^{4}, 10^{5}, 3 \times 10^{5}$ and $10^{6}$, respectively. In each case, there is a maximum $\dot{m}$ up to which an ADAF is allowed, which is referred to as $\dot{m}_{\text {crit }}$. 


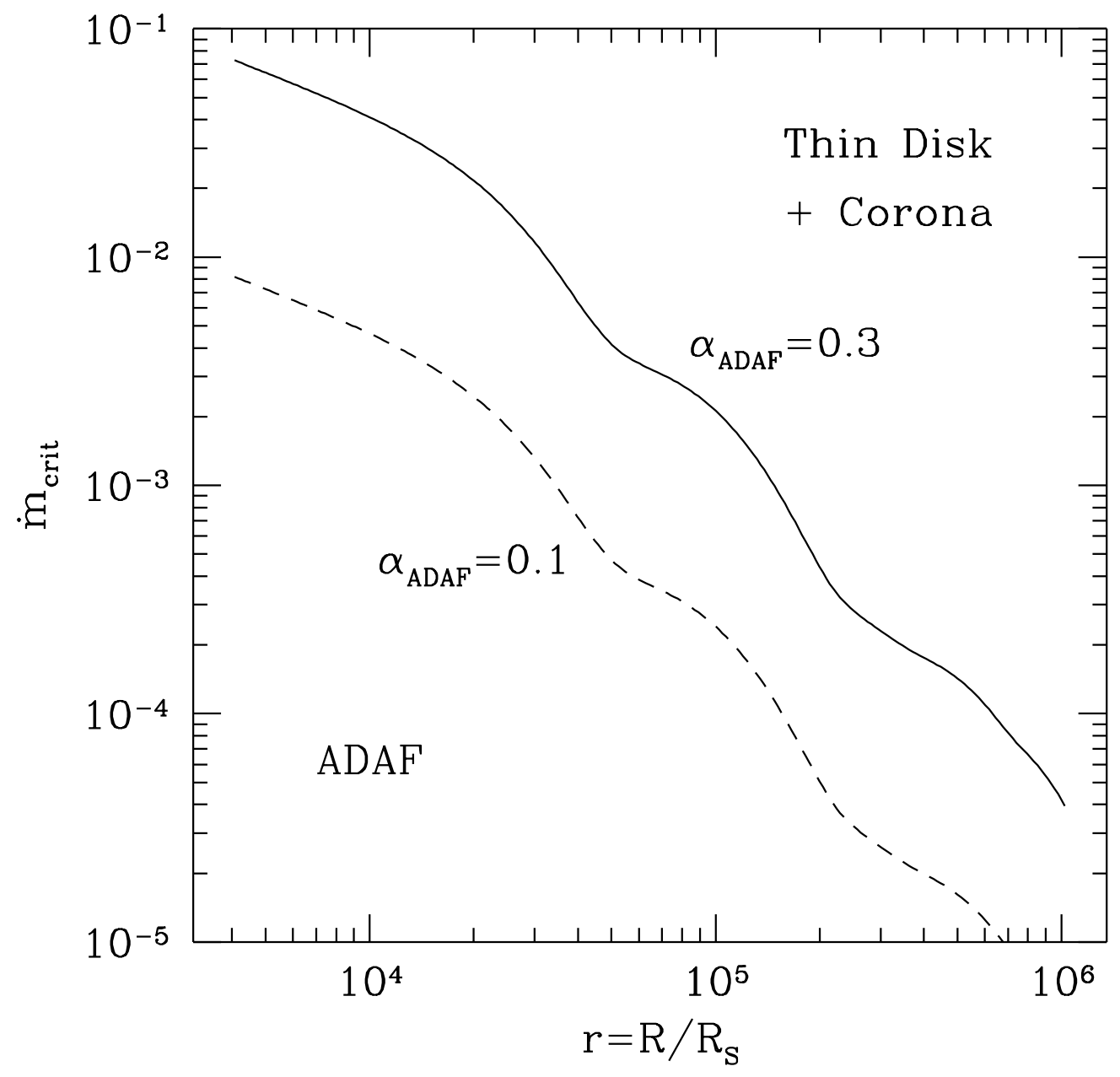

Fig. 3. - Shows the critical mass accretion rate $\dot{m}_{\text {crit }}$ as a function of radius $r$ in an ADAF for $\alpha_{\mathrm{ADAF}}=0.1$ (dashed line) and $\alpha_{\mathrm{ADAF}}=0.3$ (solid line). An ADAF is allowed only below and to the left of these lines. 


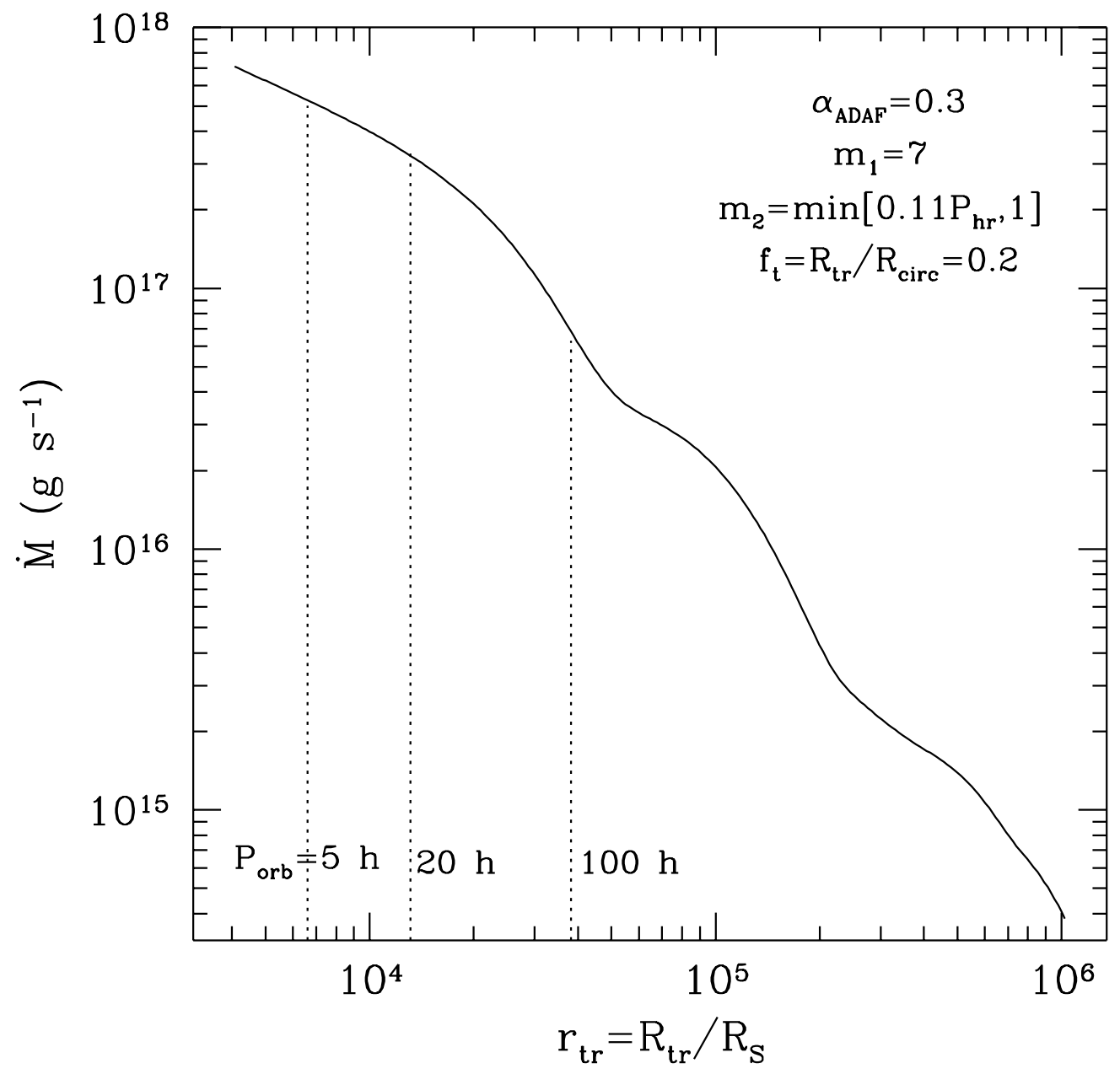

Fig. 4.- Shows the prescription we propose for the transition radius $r_{\mathrm{tr}}$. The plot corresponds to a LMBHB with $\alpha_{\mathrm{ADAF}}=0.3, m_{1}=7, m_{2}=\min \left[0.11 P_{\mathrm{hr}}, 1\right]$, and $f_{t} \equiv R_{\mathrm{tr}} / R_{\text {circ }}=0.2$. The vertical dotted lines show the location of the transition radius, as determined from stream dynamics, for three different orbital periods. The solid line is the maximum accretion rate above which an ADAF is not allowed. Equivalently, for a given accretion rate $\dot{M}$, the solid line shows the maximum radius $r_{\max }$ to which the ADAF can extend. 


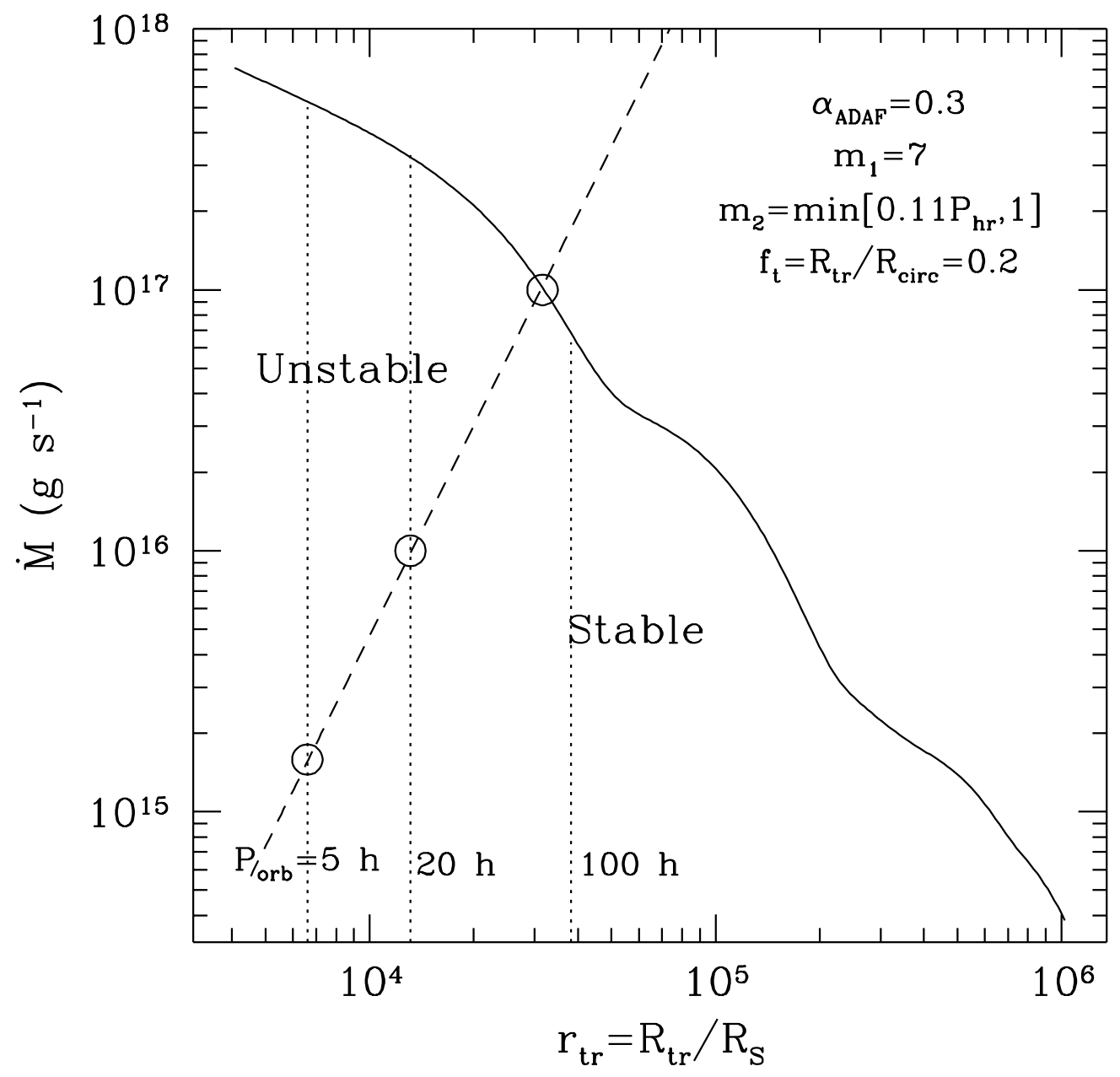

Fig. 5.- Similar to Fig. 4 but shows in addition the limit on the transition radius for stability of the outer disk (dashed line). For a given $\dot{M}$, the disk is stable if the transition radius is to the right of the dashed line, and unstable if it is to the left. The three open circles show cases of marginal stability for three different values of $P_{\text {orb }}$ and $r_{\mathrm{tr}}$. (See the text for details.) 


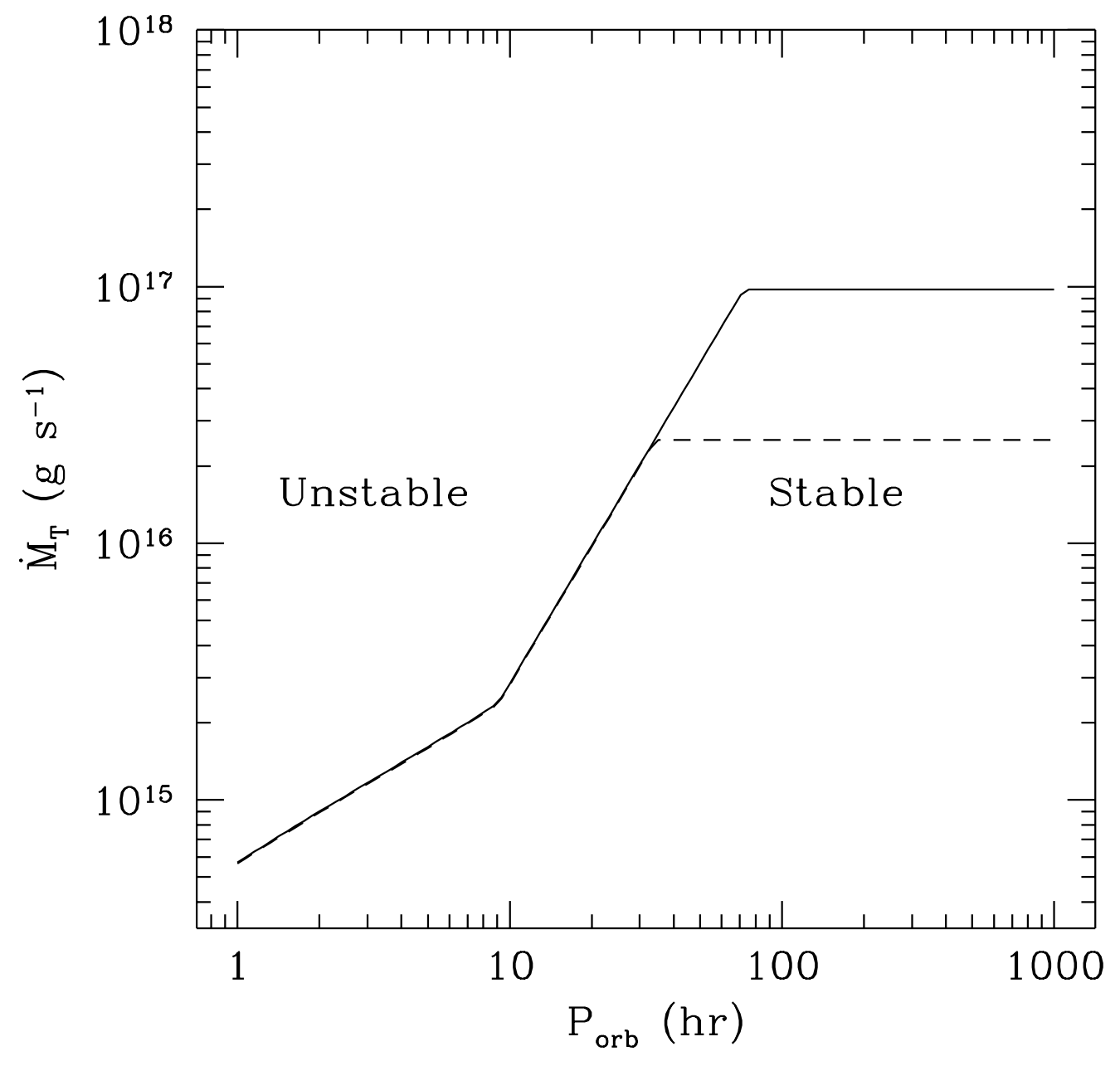

Fig. 6. - Stability criterion (solid line) for a LMBHB with the following parameters: $\alpha_{\mathrm{ADAF}}=0.3, m_{1}=7$, $m_{2}=\min \left[0.11 P_{\mathrm{hr}}, 1\right]$ and $f_{t} \equiv R_{\mathrm{tr}} / R_{\text {circ }}=0.2$. A LMBHB of a given orbital period is unstable if it accretes mass at a rate larger than that indicated by the solid line. The dashed line shows the same stability criterion if $\alpha_{\mathrm{ADAF}}=0.1$. The parameter space for stable LMBHBs is reduced in that case. 

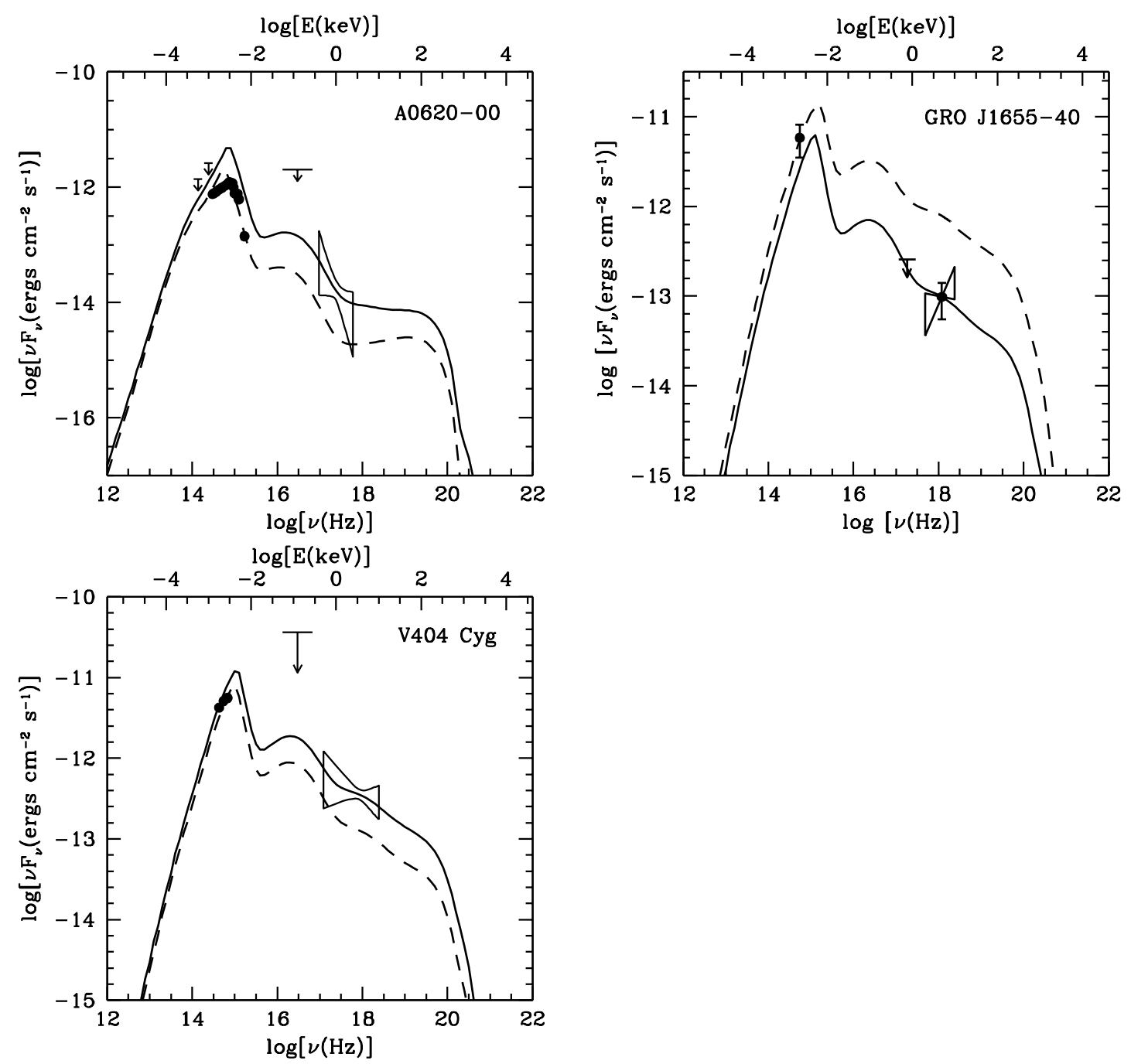

Fig. 7.- Model spectra of A0620-00, J1655-40, and V404 Cyg. The solid lines show models in which the accretion rate is adjusted to fit the X-ray data, and the dashed lines correspond to models in which the accretion rate is adjusted to fit the optical data. 

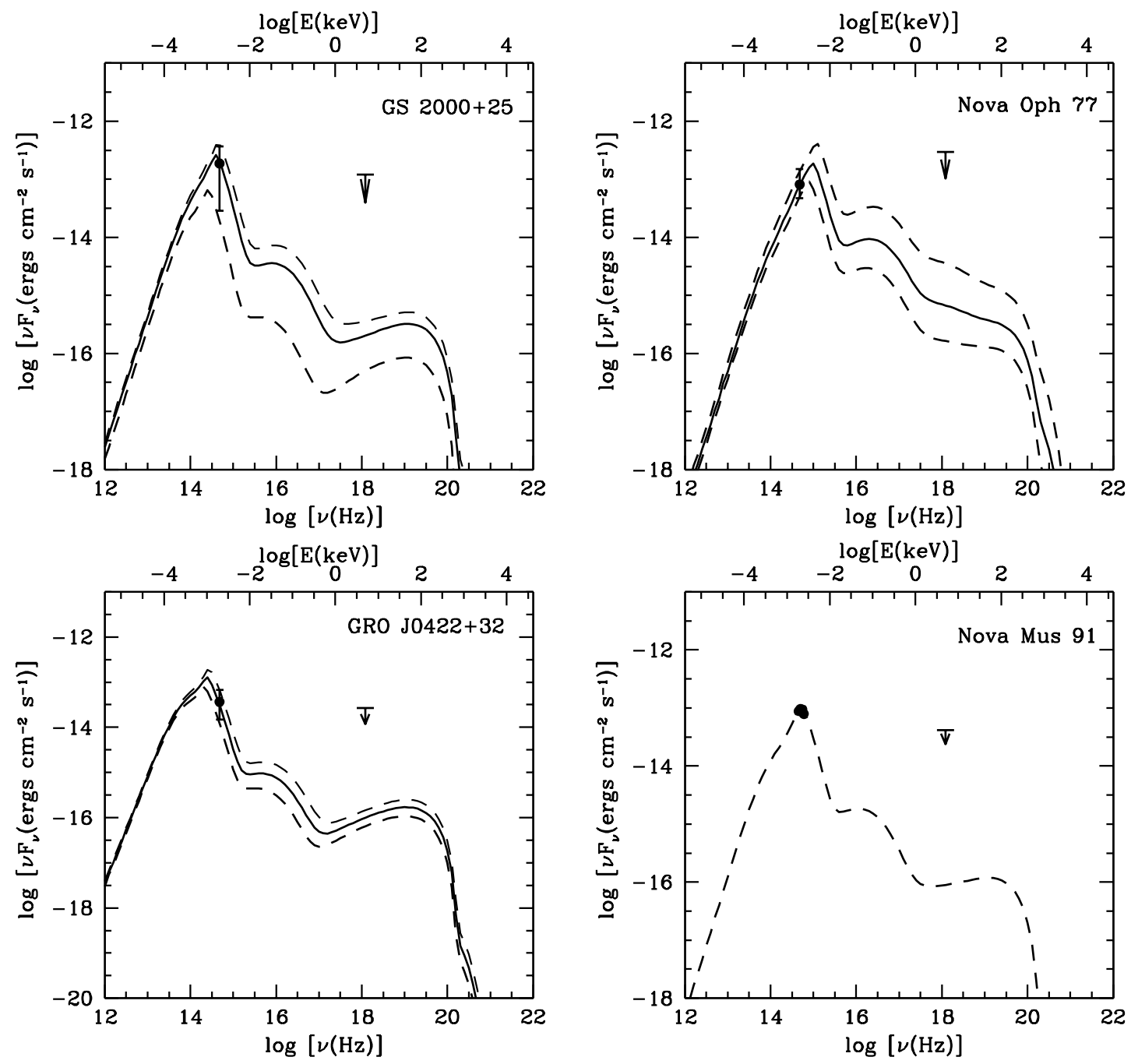

Fig. 8. - Model spectra of GS2000+25, Nova Oph 77, GRO J0422+32, and Nova Mus 91. The solid and dashed lines show models with various accretion rates which cover the range of uncertainties in the optical fluxes. All the models satisfy the X-ray upper limits. 


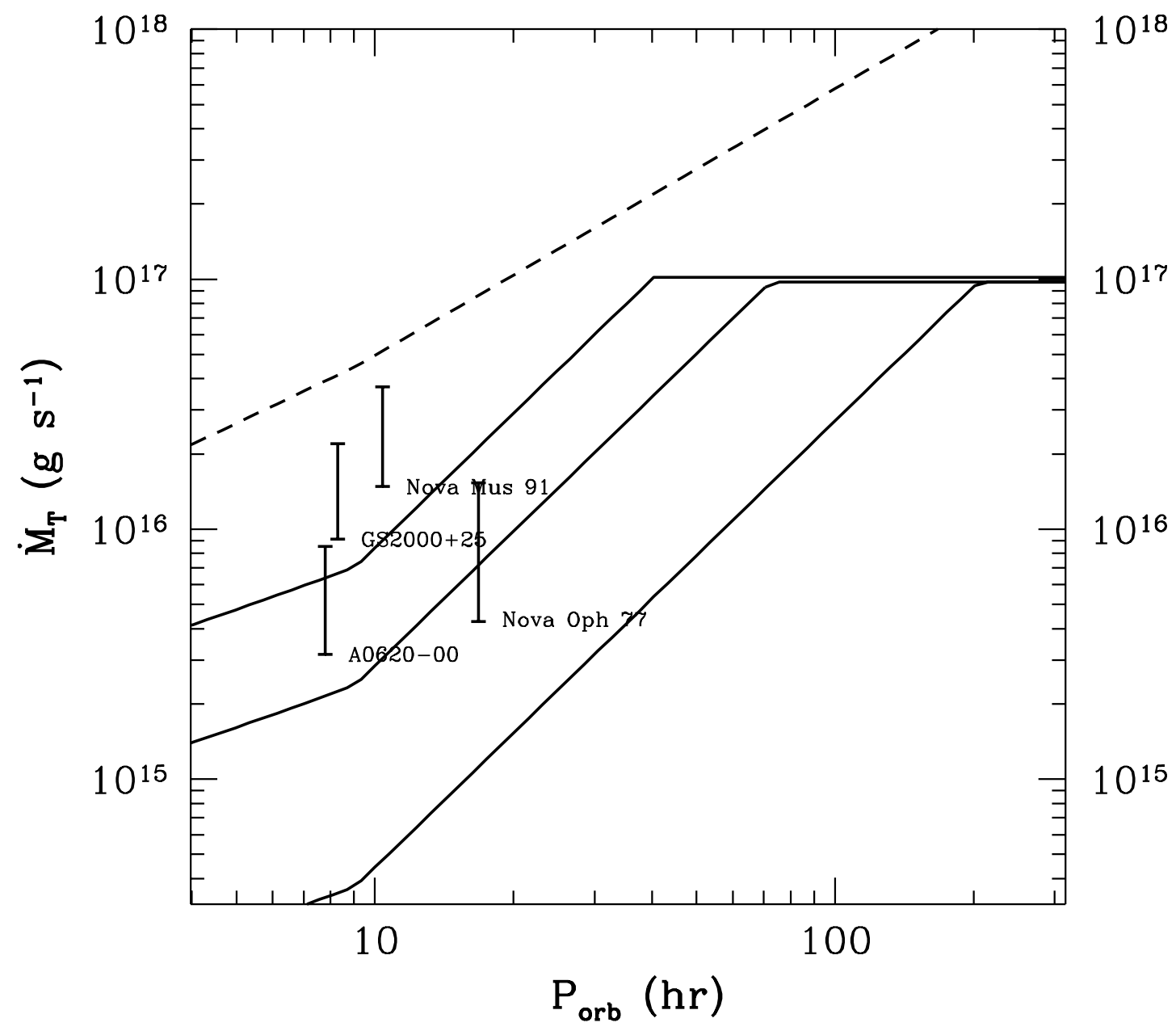

Fig. 9.- Shows the three regions of stability for a LMBHB with $\alpha_{\mathrm{ADAF}}=0.3, m_{1}=7$, and $m_{2}=$ $\min \left[0.11 P_{\mathrm{hr}}, 1\right]$. The three solid lines correspond, from above, to $f_{t}=0.3,0.2,0.1$. A system which lies below these lines is stable and will be a faint persistent LMBHB. The dashed line corresponds to the stability criterion of van Paradijs (1996). A system that is above this line is also stable and will be a bright persistent LMBHB. Systems that lie in between the dashed and solid lines are unstable and will be seen as BH SXTs. The error bars correspond to estimates of $\dot{M}_{T}$ of four BH SXTs. These systems lie in the unstable zone, as required, provided $f_{t} \lesssim 0.25-0.2$. The remaining three BH SXTs are not plotted as their masses are different from the choices made here. 

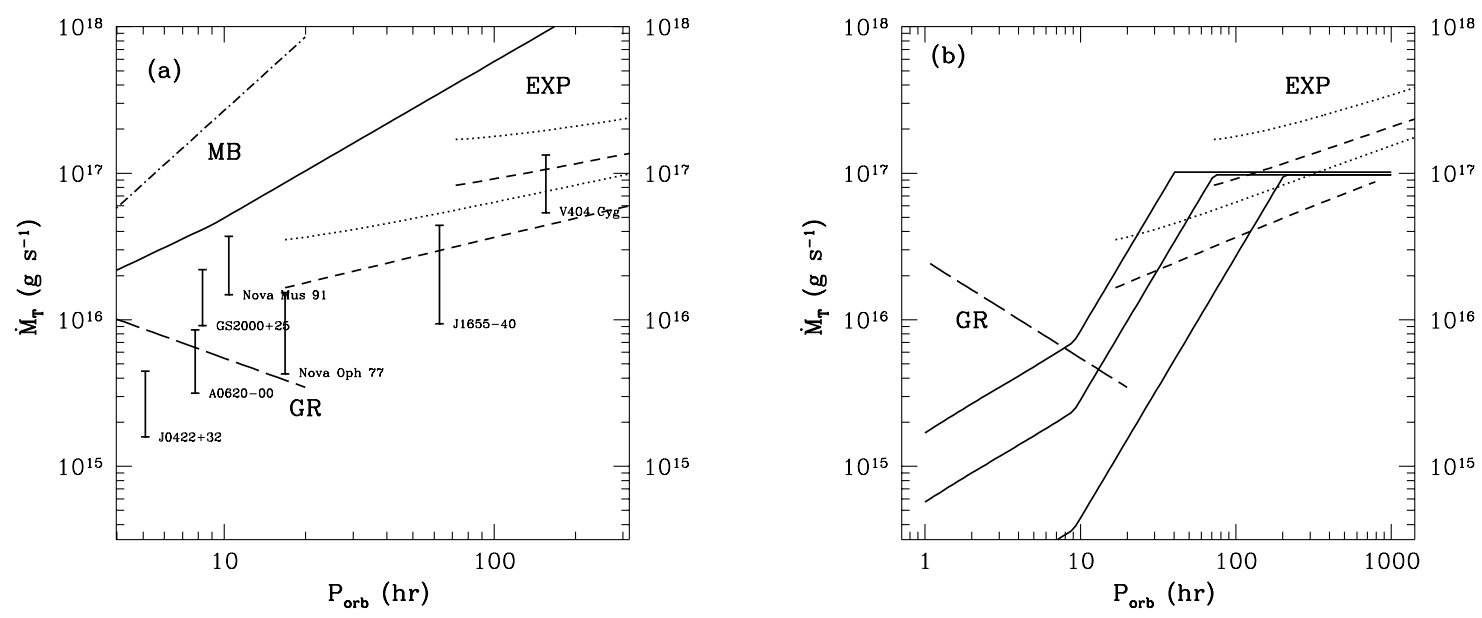

Fig. 10.- (a) A comparison of the estimates of $\dot{M}_{\text {tot }}$ (errobars) for the seven BH SXTs listed in Table 1 with the mass transfer rates predicted by binary evolution models. At low orbital periods, $P_{\text {orb }}<20 \mathrm{hr}$, predictions for gravitational radiation driven mass transfer (GR, long dashed line) and magnetic braking driven mass transfer ( $\mathrm{MB}$, dotted-dashed line) are shown. At longer orbital periods, evolutionary tracks corresponding to mass transfer driven by secondary expansion (EXP) are shown. Four tracks, corresponding to secondary initial masses of $m_{2}=1$ (dashed lines) and 1.5 (dotted lines), and two different initial orbital periods $\left(P_{\text {orb }}=17 \mathrm{hr}\right.$ and $72 \mathrm{hr}$ ) are shown. The solid line corresponds to the stability criterion of van Paradijs (1996). (b) Same as (a), except that the mass transfer rates predicted by binary evolution models (GR and EXP) are compared to the stability criteria shown in Fig. 9. The region below the solid lines is stable. Note that some segments of the evolutionary tracks lie in the stable zone. Systems that correspond to these stable segments would appear as faint persistent LMBHBs. 


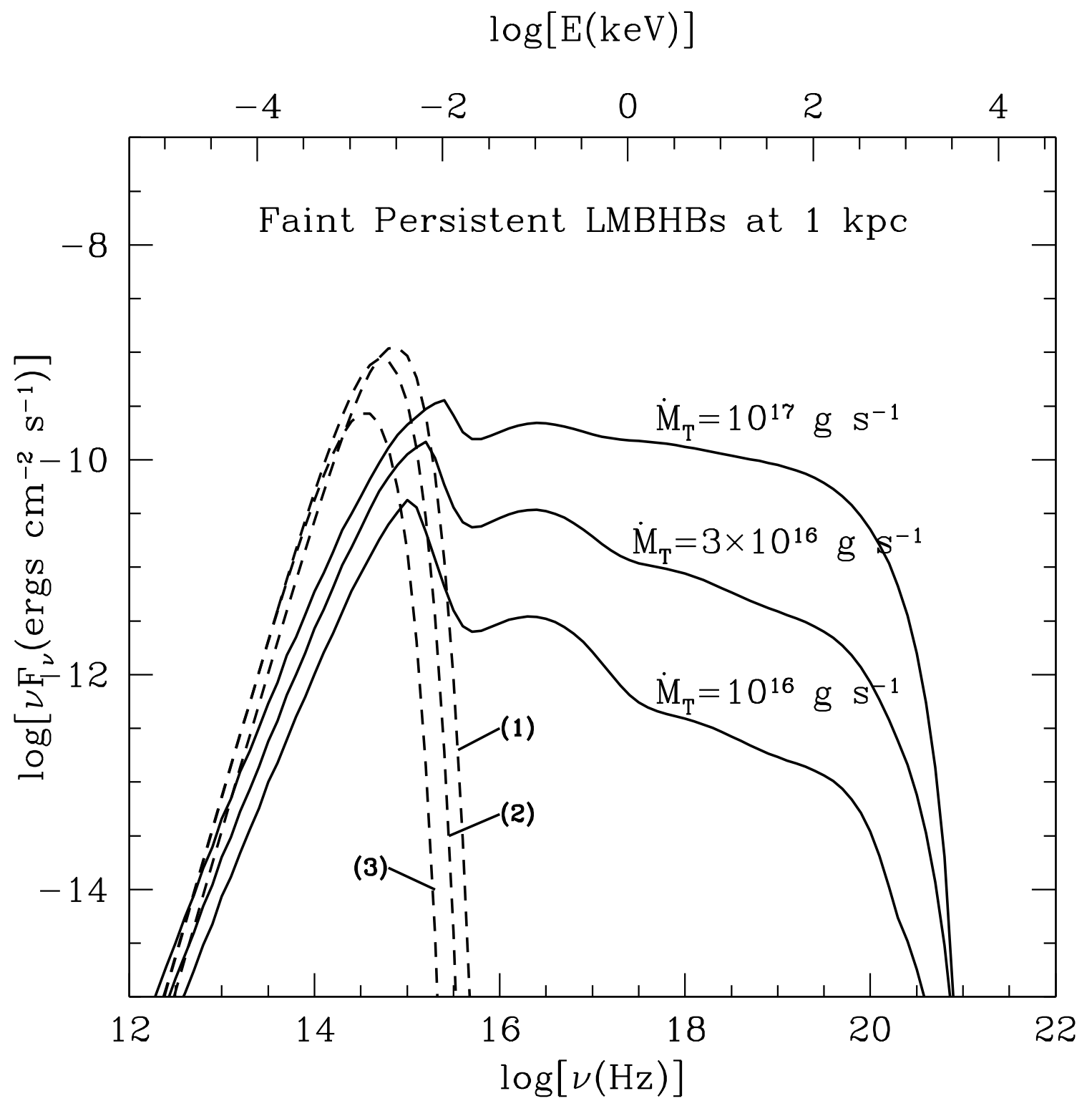

Fig. 11. - Model spectra of faint persistent LMBHBs corresponding to three mass accretion rates (solid lines). The models assume that the sources are at a distance of $1 \mathrm{kpc}$ and take $\alpha_{A D A F}=0.3, \beta=0.5$ and $m_{1}=7$. The dashed lines show spectra of the secondary stars in the long-period SXTs, $4 \mathrm{U}$ 1543-47 (1), GRO J1655-40 (2) and V404 Cyg (3). Faint persistent LMBHBs are expected to have similar secondaries. Note that the optical emission from the accretion flow is likely to be swamped by the emission from the secondaries. 